\title{
PTF11mnb: First analog of supernova 2005bf
}

\section{Long-rising, double-peaked supernova Ic from a massive progenitor ${ }^{\star}$}

\author{
F. Taddia ${ }^{1}$, J. Sollerman ${ }^{1}$, C. Fremling ${ }^{1,5}$, E. Karamehmetoglu ${ }^{1}$, R. M. Quimby ${ }^{2,3}$, A. Gal-Yam ${ }^{4}$, O. Yaron ${ }^{4}$, \\ M. M. Kasliwal ${ }^{5}$, S. R. Kulkarni ${ }^{5}$, P. E. Nugent ${ }^{6,7}$, G. Smadja ${ }^{8}$, and C. Tao ${ }^{9,10}$ \\ 1 The Oskar Klein Centre, Department of Astronomy, Stockholm University, AlbaNova, 10691 Stockholm, Sweden \\ e-mail: francesco.taddia@astro.su.se \\ 2 Department of Astronomy, San Diego State University, San Diego, CA 92182, USA \\ 3 Kavli IPMU (WPI), UTIAS, The University of Tokyo, Kashiwa, 277-8583 Chiba, Japan \\ ${ }^{4}$ Benoziyo Center for Astrophysics, Weizmann Institute of Science, 76100 Rehovot, Israel \\ 5 Cahill Center for Astrophysics, California Institute of Technology, Pasadena, CA 91125, USA \\ 6 Astronomy Department, University of California at Berkeley, Berkeley, CA 94720, USA \\ 7 Lawrence Berkeley National Laboratory, 1 Cyclotron Road, MS 50B-4206, Berkeley, CA 94720, USA \\ 8 Université de Lyon 1, Villeurbanne; CNRS/IN2P3, Institut de Physique Nucléaire de Lyon, 69622 Lyon, France \\ 9 Tsinghua Center for Astrophysics, Tsinghua University, 100084 Beijing, PR China \\ 10 Centre de Physique des Particules de Marseille, Aix-Marseille Université, CNRS/IN2P3, 163 avenue de Luminy, Case 902, \\ 13288 Marseille Cedex 09, France
}

Received 10 October 2016 / Accepted 12 October 2017

\begin{abstract}
Aims. We study PTF11mnb, a He-poor supernova (SN) whose light curves resemble those of SN 2005bf, a peculiar double-peaked stripped-envelope (SE) SN, until the declining phase after the main peak. We investigate the mechanism powering its light curve and the nature of its progenitor star.

Methods. Optical photometry and spectroscopy of PTF11mnb are presented. We compared light curves, colors and spectral properties to those of SN 2005bf and normal SE SNe. We built a bolometric light curve and modeled this light curve with the SuperNova Explosion Code (SNEC) hydrodynamical code explosion of a MESA progenitor star and semi-analytic models.

Results. The light curve of PTF11mnb turns out to be similar to that of SN 2005bf until 50 d when the main (secondary) peaks occur at $-18.5 \mathrm{mag}$. The early peak occurs at $\sim 20 \mathrm{~d}$ and is about 1.0 mag fainter. After the main peak, the decline rate of PTF11mnb is remarkably slower than what was observed in SN 2005bf, and it traces well the ${ }^{56} \mathrm{Co}$ decay rate. The spectra of PTF11mnb reveal a SN Ic and have no traces of He unlike in the case of SN Ib 2005bf, although they have velocities comparable to those of SN $2005 \mathrm{bf}$. The whole evolution of the bolometric light curve is well reproduced by the explosion of a massive $\left(M_{e j}=7.8 M_{\odot}\right)$, He-poor star characterized by a double-peaked ${ }^{56} \mathrm{Ni}$ distribution, a total ${ }^{56} \mathrm{Ni}$ mass of $0.59 M_{\odot}$, and an explosion energy of $2.2 \times 10^{51}$ erg. Alternatively, a normal SN Ib/c explosion $\left(M\left({ }^{56} \mathrm{Ni}\right)=0.11 M_{\odot}, E_{K}=0.2 \times 10^{51} \mathrm{erg}, M_{e j}=1 M_{\odot}\right)$ can power the first peak while a magnetar, with a magnetic field characterized by $B=5.0 \times 10^{14} \mathrm{G}$, and a rotation period of $P=18.1 \mathrm{~ms}$, provides energy for the main peak. The early $g$-band light curve can be fit with a shock-breakout cooling tail or an extended envelope model from which a radius of at least $30 R_{\odot}$ is obtained.

Conclusions. We presented a scenario where PTF11mnb was the explosion of a massive, He-poor star, characterized by a doublepeaked ${ }^{56} \mathrm{Ni}$ distribution. In this case, the ejecta mass and the absence of He imply a large ZAMS mass $\left(\sim 85 M_{\odot}\right)$ for the progenitor, which most likely was a Wolf-Rayet star, surrounded by an extended envelope formed either by a pre-SN eruption or due to a binary configuration. Alternatively, PTF11mnb could be powered by a SE SN with a less massive progenitor during the first peak and by a magnetar afterward.
\end{abstract}

Key words. supernovae: general - supernovae: individual: PTF11mnb, SN 2005bf, iPTF15dtg

\section{Introduction}

The majority of stripped-envelope (SE) supernovae ( $\mathrm{SNe}$ ) share rather similar light-curve properties (see, e.g., Drout et al. 2011; Cano 2013; Bianco et al. 2014; Taddia et al. 2015, 2017; Lyman et al. 2016; Prentice et al. 2016) with typical rise times of 15-25 days in the optical, slower decline, and peak magnitudes between -17 and -18 mag. Most of these SNe are inferred to have ejected $2-4 M_{\odot}$ with energies of a few $10^{51} \mathrm{erg}$ and

\footnotetext{
* Photometric tables are only available at the CDS via anonymous ftp to cdsarc.u-strasbg. fr (130.79.128.5) or via http://cdsarc.u-strasbg.fr/viz-bin/qcat?J/A+A/609/A106
}

${ }^{56} \mathrm{Ni}$ masses between $0.15-0.20 M_{\odot}$. Assuming these $\mathrm{SNe}$ gave birth to compact remnants with the mass of a neutron star, their rather modest ejecta masses suggest that $\mathrm{SE} \mathrm{SNe}$ arise from relatively low-mass $\left(M_{\text {ZAMS }} \lesssim 15 M_{\odot}\right.$ ) progenitors, which then must have been stripped of their $\mathrm{H} / \mathrm{He}$ envelopes by a companion star (e.g., Yoon et al. 2010; Eldridge et al. 2013; Lyman et al. 2016).

A few SE SN events show clearly different properties with light curves characterized by a more complex morphology and different timescales. Two examples are SN $2011 \mathrm{bm}$ (Valenti et al. 2012) and iPTF15dtg (Taddia et al. 2016a), which are characterized by longer rise times $(\sim 30-40 \mathrm{~d})$ and broader light curves. iPTF15dtg (and perhaps SN 2011bm) also exhibits 
an early declining phase in the optical light curves (Taddia et al. 2016a). These SNe may have large ejecta masses $\left(\sim 10 M_{\odot}\right)$ compared to normal SE SNe and might come from single massive stars. Another unusual SE SN is the double-peaked SN 2005bf. This transient was discovered by Monard et al. (2005) and became one of the most peculiar SE SNe ever observed. SN 2005bf has remained unique until the discovery of PTF11 mnb, which we present in this paper.

Therefore, we start by reviewing the main properties of SN 2005bf as presented in the literature.

Anupama et al. (2005) reported optical photometry and spectroscopy around and post peak for SN 2005bf. This SN revealed a Type Ib (helium rich) spectrum at peak, which occurred unusually late for a SE SN ( 40 d). The peak bolometric magnitude of -18 mag was consistent with those of luminous SNe Ibc. Anupama et al. (2005) observed that the He I lines were less blueshifted than the Fe lines, and reported traces of $\mathrm{H}$ lines at $15000 \mathrm{~km} \mathrm{~s}^{-1}$. These authors suggested that SN 2005bf was the explosion of a massive $\mathrm{He}$ star with some $\mathrm{H}$ left.

Tominaga et al. (2005) reported that the light curve of SN 2005bf actually had two maxima: the first at $\sim 20 \mathrm{~d}$ and the main (secondary) peak at $40 \mathrm{~d}$. After the second peak the light curve faded rapidly. Tominaga et al. (2005) noted that the He I lines strengthened and their velocities increased with time. A double-peaked ${ }^{56} \mathrm{Ni}$ distribution was proposed to explain the two maxima with a small amount at high velocity and most of the ${ }^{56} \mathrm{Ni}$ at low velocity. The fast decline after peak could be due to $\gamma$-rays escaping from low-density regions. The evolution of the He lines was then explained by enhanced $\gamma$-ray deposition in the He layer with time, as these $\gamma$-rays were leaking out from the core. From their models, Tominaga et al. (2005) estimated a large ejecta mass $\left(\sim 6-7 M_{\odot}\right)$, kinetic energy of $(1.0-1.5) \times 10^{51} \mathrm{ergs}$, and a large ${ }^{56} \mathrm{Ni}$ mass $\left(\sim 0.32 M_{\odot}\right)$. The progenitor was claimed to be a WN star, whose double-peaked ${ }^{56} \mathrm{Ni}$ distribution could possibly be due to jets that did not reach the He layer.

Folatelli et al. (2006) presented observations of SN 2005bf covering the first $\sim 100$ days after discovery. Their spectroscopic observations revealed that SN 2005bf exhibited increasingly stronger He lines. Furthermore, high velocity absorption lines were observed during the initial peak along with lower velocity line components (see also Parrent et al. 2007). The scenario favored by Folatelli et al. (2006) was an energetic and asymmetric explosion of a massive $\left(8.3 M_{\odot}\right) \mathrm{WN}$ star almost completely stripped of its $\mathrm{H}$ envelope. Folatelli et al. (2006) attributes high velocity features, early spectrum, and the existence of the first peak to a polar explosion containing only part of the total mass; that early explosion was then followed by the explosion of the rest of the star, which produced the main peak and helium-rich spectra.

Maeda et al. (2007) presents nebular spectra ( 300 d) and late-time photometry of SN 2005bf. The emission line analysis reveals a blueshift compatible with a blob or a unipolar jet (or self- absorption within the ejecta) containing only tenths of a solar mass of ejecta and a small amount $\left(0.02-0.06 M_{\odot}\right)$ of ${ }^{56} \mathrm{Ni}$. The late-time photometry sets an upper limit of $0.08 M_{\odot}$ for the ${ }^{56} \mathrm{Ni}$ mass, in apparent contradiction to the high value derived from the main peak. To explain this discrepancy and the fast decline of the light curve after peak, Maeda et al. (2007) suggested an alternative scenario where the powering source of SN 2005bf is a magnetar. The strong asymmetry of the explosion of SN 2005bf was also confirmed by the spectropolarimetric observations by Maund et al. (2007) and Tanaka et al. (2009).
In summary, SN 2005bf showed the following peculiarities: a) a unique double peak in the light curve, with the first maximum occurring at the same phase $(\sim 20 \mathrm{~d})$ and with the same absolute magnitude of a regular SN Ibc, while the main peak occurred relatively late ( $\sim 40 \mathrm{~d})$; b) a fast decline post peak and a very low optical luminosity at late epochs; c) increasingly stronger and faster He lines; d) multiple velocity components for some of the lines.

In this paper, we present PTF11mnb, the first SN 2005bflike event. We use SN 2005bf-like to mean a SE SN with a first peak similar to that of a normal $\mathrm{SN} \mathrm{Ib/c}$, followed by a brighter peak occurring on a longer timescale. PTF11mnb was a He-poor (Type Ic) SN whose pre-main peak optical light curves closely resemble those of SN 2005bf. Both SNe show a double peak at similar phases and absolute magnitudes, but PTF11mnb declines more slowly after the main maximum. Furthermore, PTF11mnb never shows He in the spectrum and thus never becomes a Type Ib. For SN PTF11mnb, we suggest a scenario in which the progenitor was a massive single star with a doublepeaked ${ }^{56} \mathrm{Ni}$ distribution powering the $\mathrm{SN}$ rather than a magnetar, even though we cannot exclude the presence of a central engine.

The structure of the paper is as follows: In Sect. 2 we describe the discovery, observations, and data reduction; in Sect. 3 we present the host galaxy. Section 4 includes the analysis of the SN light curves, whereas Sect. 5 includes that of the SN spectra. In Sect. 6 we build and model the bolometric light curve of PTF11mnb. The main results are discussed in Sect. 7, whereas our conclusions are given in Sect. 8.

\section{Observations and data reduction}

The Palomar Transient Factory (Rau et al. 2009; Law et al. 2009) first detected PTF11mnb at RA $=00: 34: 13.25$ and Dec $=+02: 48: 31.4$ (J2000.0) on JD 2455804.857 (Aug. 31 2011) $(g=21.07 \pm 0.31 \mathrm{mag})$ using the 48 -inch Samuel Oschin telescope (P48) at Palomar Observatory, equipped with the 96 Mpixel mosaic camera CFH12K (Rahmer et al. 2008). The SN was not detected on JD 2455803.824 (i.e., 1.033 days before first detection) at limiting magnitude $g \geq 21.41 \mathrm{mag}$. In the following, we adopt the average between the epochs of the last non-detection and discovery as the explosion date $\left(t_{\text {explo }}=\right.$ JD $\left.2455804.341 \pm 0.516\right)$. Throughout the paper we express the phase in SN rest-frame days since explosion.

PTF11mnb was observed with P48 in the $g$ band until $\sim 60 \mathrm{~d}$ and until $\sim 50$ days in the $r$ band. The P48 photometry was reduced with the FPipe pipeline presented by Fremling et al. (2016), which gives very similar results to those obtained using the standard Palomar Transient Factory Image Differencing and Extraction (PTFIDE) pipeline (Masci et al. 2017). The FPipe pipeline subtracts the host-galaxy template and performs point spread function (PSF) photometry on each SN image.

We also made use of the Palomar 60-inch telescope (P60; Cenko et al. 2006), with which we observed the SN in Bgri bands, starting at $\sim 20 \mathrm{~d}$ with the $r$ band (Bgi-band coverage started from $\sim 40 \mathrm{~d}$ ). The $\mathrm{SN}$ was detected for the last time at $\sim 140$ days with P60. A P60 composite Bgr-band image of PTF11mnb and its host galaxy from 28 October 2011 is shown in the top panel of Fig. 1. The P60 images were also reduced with FPipe, making use of Sloan Digital Sky Survey (SDSS; Ahn et al. 2014) images as templates. We used the SDSS stars in the SN field to calibrate the P60 photometry. The final light curves are presented after combining magnitudes obtained on the same night. We summarize all the photometric observations in Table 1. 

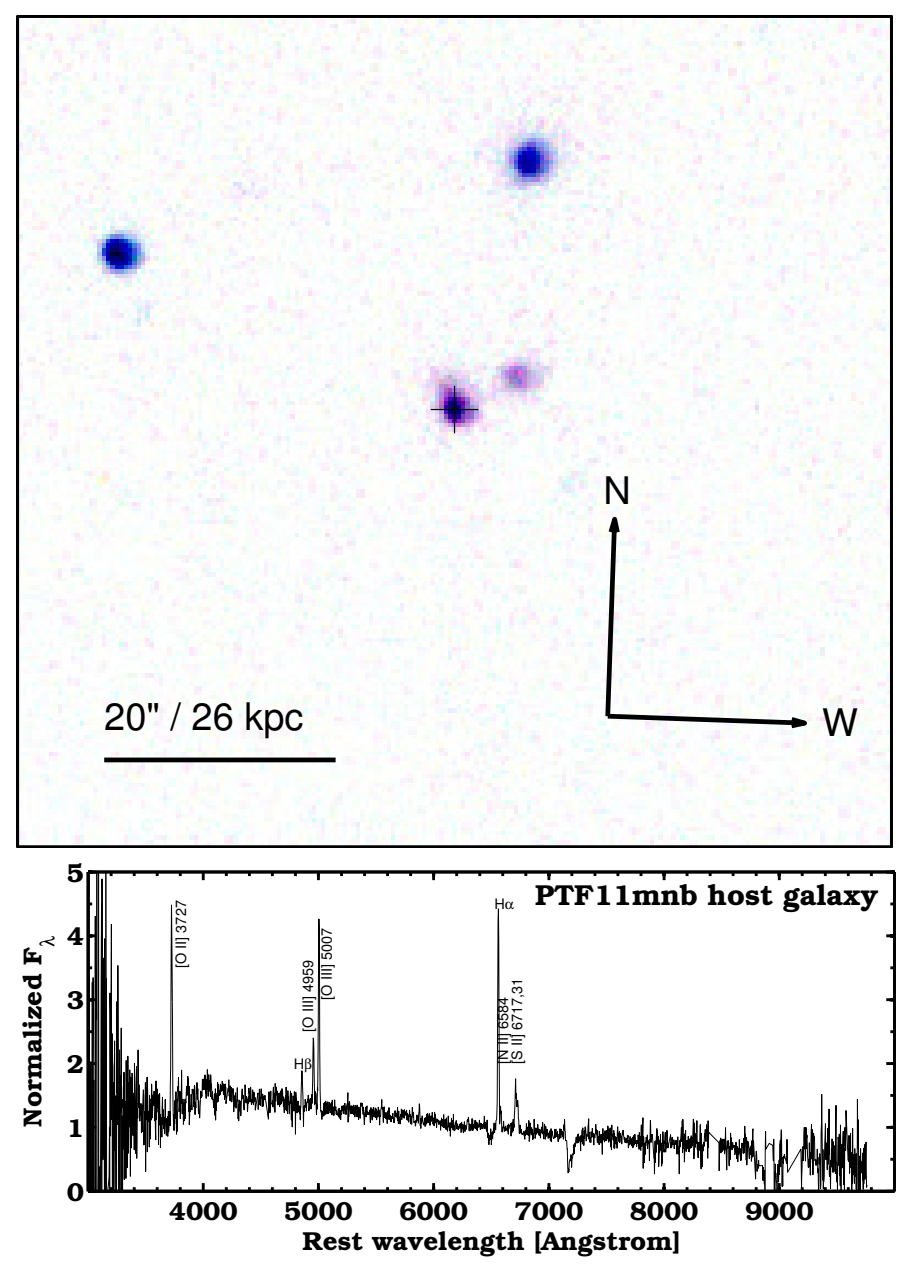

Fig. 1. Top panel: PTF11mnb (indicated by a black cross) and its host galaxy in a composite Bgr image (with inverted colors) from P60 observations taken on 28 October 2011. The orientation of the image is indicated in the bottom-right corner, whereas the scale is shown in the bottom left. Bottom panel: TNG spectrum of the host galaxy of PTF11mnb obtained in 2016. The main emission lines are identified.

We present five optical spectra covering the epochs between one and five months after explosion. These spectra were obtained with four different telescopes: Keck I equipped with the Low Resolution Imaging Spectrometer (LRIS; Oke et al. 1995), the Palomar 200-inch Hale Telescope (P200) with the Double Spectrograph (DBSP; Oke \& Gunn 1982), the Kitt Peak National Observatory 4-m telescope (KPNO4m) with the Ritchey-Chretien Spectrograph (RC spec), and the University of Hawai'i 88-inch $(2.2 \mathrm{~m})$ telescope (UH88) with the Supernova Integrated Field Spectrograph (SNIFS). Spectral reductions were carried out with all the standard procedures, including wavelength calibration via an arc lamp, and flux calibration with a spectrophotometric standard star. In 2016 we also obtained a host-galaxy spectrum with the Telescopio Nazionale Galileo (TNG) equipped with the Device Optimized for the LOw RESolution (DOLORES). This host-galaxy spectrum is shown in the bottom panel of Fig. 1. Table 2 shows our spectral log.

\section{Host galaxy}

The host galaxy of PTF11mnb is named SDSS J003413.34+ 024832.9. We determined its redshift to be $z=0.0603 \pm 0.0001$ from the Gaussian fits of the $\mathrm{H} \alpha$ and [O III] $\lambda 5007$ host-galaxy emission lines superimposed on the SN spectra (see Sect. 5), as well as from the host-galaxy spectrum obtained in 2016 (see Fig. 1). This redshift corresponds to a luminosity distance $D_{L}=268.5 \mathrm{Mpc}$ and distance modulus $\mu=37.14 \mathrm{mag}$ when WMAP 5-yr cosmological parameters (Komatsu et al. 2009) are assumed.

Given the absence of any narrow Na I D absorption line at the host-galaxy redshift, we assume that no host extinction affected PTF11mnb. The Galactic extinction in the Bgri bands is $A_{B}=0.067 \mathrm{mag}, A_{g}=0.061 \mathrm{mag}, A_{r}=0.042 \mathrm{mag}$, and $A_{i}=0.031 \mathrm{mag}$ (Schlafly \& Finkbeiner 2011).

SDSS J003413.34+024832.9 has integrated magnitudes of $M_{g}=-17.8 \mathrm{mag}, M_{r}=-18.0 \mathrm{mag}$, and $M_{i}=-18.3 \mathrm{mag}$. Based on these absolute magnitudes, a global metallicity of $12+$ $\log (\mathrm{O} / \mathrm{H})=8.29\left(Z / Z_{\odot}=0.4\right.$, where $Z_{\odot}=8.69$, Asplund et al. $2009)$ is inferred from the luminosity-color-metallicity relation by Sanders et al. (2013), or $Z / Z_{\odot}=0.25$ if we make use of the luminosity-metallicity relation by Arcavi et al. (2010).

Since PTF11mnb sits on a bright H II region, we could measure the host emission-line fluxes at the exact SN position from its spectra. Using the high signal-to-noise Keck spectrum, we derived the line ratios needed for the O3N2 method by Pettini \& Pagel (2004), which resulted in a metallicity of $12+\log (\mathrm{O} / \mathrm{H})=8.29 \pm 0.20\left(Z / Z_{\odot}=0.40\right)$. This value is lower than that for most of the normal SNe Ic (e.g., Sanders et al. 2012) and more similar to that of the explosion site of the peculiar SN Ic iPTF15dtg (Taddia et al. 2016a).

We also noticed that the host galaxy of SN 2005bf shows larger metal abundances. From a Sloan Digital Sky Survey (SDSS) spectrum of an HII region in a spiral arm of the host of SN 2005bf, only marginally closer to the host center than SN 2005bf itself, we measured an O3N2 oxygen abundance of $8.76 \pm 0.14$, which is about solar (Asplund et al. 2009).

We summarize the main host-galaxy properties in Table 3.

\section{Light curves}

In Fig. 2 we present the Bgri light curves of PTF11mnb. The first detection in $g$ band occurred at $\sim-15.63 \mathrm{mag}$. For four days (first three epochs) the light curve in the $g$ band shows an almost constant value (within the errors). Thereafter, the SN was observed rising faster in $g$ band and imaged in the $r$ band with P48. The $r$-band light curve shows a steep rise of $\sim 1.3 \mathrm{mag}$ from $+5.2 \mathrm{~d}$ to $+17.4 \mathrm{~d}$. This is followed by a flatter phase until 21.3 days. This phase is also seen in the $g$ band as an early peak/plateau between $\sim 20 \mathrm{~d}$ and $27 \mathrm{~d}$. Following this early peak, both $g$ and $r$ band rise to the main peak, which occurs at $+46.3 \mathrm{~d}$ and $+52.2 \mathrm{~d}$, respectively. PTF11mnb was also observed in $B$ and $i$ band starting from $+36 \mathrm{~d}$. The $B$ - and $i$-band peaks occurred at $+41.9 \mathrm{~d}$ and $+48.1 \mathrm{~d}$, respectively. We determined the maxima by fitting the light curves with low-order polynomials, marked in Fig. 2 by dashed lines.

The main peaks of PTF11mnb are very broad (especially in the redder bands) and are characterized by $\Delta m_{15}=0.48,0.53$, 0.16 , and 0.24 mag in $B, g, r$, and $i$, respectively.

After an almost linear decline that lasts until $+106 \mathrm{~d}$ in $r$ and $i$ band and $+93 \mathrm{~d}$ in the $g$ band, the late light curves show a shallow rebrightening ( 0.1 mag in $r$ and $i$ ) that peaks at $\sim 115 \mathrm{~d}$ in $g$ band and at $\sim 120 \mathrm{~d}$ in $r$ and $i$ band. The last detection occurs after this shallow bump at $+140 \mathrm{~d}(r$ and $i)$ and $+132 \mathrm{~d}(\mathrm{~g})$.

The comparison to the light curves of SN 2005bf reveals how these two events are similar, at least during the epochs up to 
Table 1. Optical photometry of PTF11mnb.

\begin{tabular}{|c|c|c|c|c|c|c|c|}
\hline $\begin{array}{l}\text { JD-2 } 455000 \\
\text { (days) }\end{array}$ & $\begin{array}{c}B \\
(\mathrm{mag})\end{array}$ & $\begin{array}{l}\text { JD-2 } 455000 \\
\text { (days) }\end{array}$ & $\begin{array}{c}g \\
(\mathrm{mag})\end{array}$ & $\begin{array}{l}\text { JD-2 } 455000 \\
\text { (days) }\end{array}$ & $\begin{array}{c}r \\
(\mathrm{mag})\end{array}$ & $\begin{array}{l}\text { JD-2 } 455000 \\
\text { (days) }\end{array}$ & $\begin{array}{c}i \\
\text { (mag) }\end{array}$ \\
\hline 842.803 & $19.096(0.060)$ & 804.857 & $21.071(0.309)$ & 809.882 & $21.146(0.195)$ & 842.801 & $18.852(0.049)$ \\
\hline 848.672 & $18.862(0.064)$ & 806.881 & $20.987(0.045)$ & 811.870 & $20.862(0.093)$ & 848.671 & $18.745(0.046)$ \\
\hline 851.679 & $19.037(0.044)$ & 807.880 & $20.948(0.041)$ & 812.845 & $20.479(0.056)$ & 851.677 & $18.669(0.004)$ \\
\hline 854.666 & $19.064(0.011)$ & 808.906 & $20.761(0.090)$ & 813.808 & $20.444(0.165)$ & 854.663 & $18.644(0.022)$ \\
\hline 857.694 & $19.081(0.014)$ & 825.748 & $19.904(0.069)$ & 821.749 & $20.017(0.026)$ & 857.692 & $18.690(0.041)$ \\
\hline 858.656 & $19.210(0.019)$ & 826.915 & $19.910(0.014)$ & 822.753 & $19.889(0.073)$ & 858.654 & $18.677(0.015)$ \\
\hline 862.656 & $19.405(0.030)$ & 827.939 & $19.816(0.042)$ & 823.750 & $19.772(0.056)$ & 862.653 & $18.721(0.024)$ \\
\hline 866.667 & $19.514(0.029)$ & 828.946 & $19.916(0.031)$ & 824.787 & $19.870(0.041)$ & 866.664 & $18.821(0.029)$ \\
\hline 869.633 & $19.667(0.124)$ & 829.925 & $19.857(0.022)$ & 824.896 & $19.645(0.017)$ & 869.630 & $18.855(0.009)$ \\
\hline 916.694 & $20.886(0.142)$ & 830.956 & $19.846(0.047)$ & 826.985 & $19.686(0.026)$ & 891.828 & $19.263(0.074)$ \\
\hline \multirow[t]{30}{*}{922.673} & $20.847(0.078)$ & 831.958 & $19.839(0.006)$ & 828.946 & $19.554(0.016)$ & 903.665 & $19.507(0.083)$ \\
\hline & & 833.768 & $19.828(0.017)$ & 838.672 & $19.199(0.060)$ & 906.794 & $19.624(0.087)$ \\
\hline & & 834.768 & $19.701(0.020)$ & 842.711 & $19.082(0.009)$ & 916.691 & $19.825(0.078)$ \\
\hline & & 835.800 & $19.551(0.015)$ & 849.636 & $18.812(0.104)$ & 922.670 & $19.680(0.053)$ \\
\hline & & 836.796 & $19.481(0.026)$ & 850.632 & $18.742(0.028)$ & 930.653 & $19.700(0.086)$ \\
\hline & & 837.819 & $19.390(0.051)$ & 851.631 & $18.797(0.007)$ & 944.688 & $19.866(0.095)$ \\
\hline & & 842.805 & $18.972(0.041)$ & 852.749 & $18.789(0.023)$ & 945.623 & $19.925(0.058)$ \\
\hline & & 848.674 & $18.821(0.054)$ & 853.737 & $18.764(0.001)$ & 952.637 & $20.148(0.103)$ \\
\hline & & 851.680 & $18.830(0.018)$ & 854.716 & $18.791(0.033)$ & & \\
\hline & & 854.668 & $18.901(0.000)$ & 862.655 & $18.694(0.016)$ & & \\
\hline & & 855.639 & $18.775(0.002)$ & 866.665 & $18.824(0.027)$ & & \\
\hline & & 856.728 & $18.775(0.011)$ & 869.632 & $18.867(0.019)$ & & \\
\hline & & 857.696 & $18.887(0.008)$ & 875.588 & $18.785(0.086)$ & & \\
\hline & & 857.729 & $18.819(0.020)$ & 891.830 & $19.364(0.146)$ & & \\
\hline & & 858.658 & $18.910(0.008)$ & 903.667 & $19.552(0.055)$ & & \\
\hline & & 858.732 & $18.850(0.027)$ & 916.692 & $19.815(0.072)$ & & \\
\hline & & 859.737 & $18.923(0.038)$ & 922.672 & $19.827(0.048)$ & & \\
\hline & & 861.654 & $18.921(0.005)$ & 923.610 & $19.700(0.055)$ & & \\
\hline & & 862.658 & $19.113(0.017)$ & 930.654 & $19.652(0.059)$ & & \\
\hline & & 862.715 & $19.058(0.029)$ & 944.690 & $19.887(0.103)$ & & \\
\hline & & 863.714 & $19.113(0.033)$ & 952.638 & $20.031(0.099)$ & & \\
\hline & & 864.729 & $19.164(0.023)$ & & & & \\
\hline & & 865.792 & $19.177(0.027)$ & & & & \\
\hline & & 866.668 & $19.296(0.023)$ & & & & \\
\hline & & 869.638 & $19.420(0.064)$ & & & & \\
\hline & & 903.670 & $20.684(0.130)$ & & & & \\
\hline & & 916.695 & $20.759(0.099)$ & & & & \\
\hline & & 923.612 & $20.618(0.101)$ & & & & \\
\hline & & 930.658 & $20.625(0.163)$ & & & & \\
\hline & & 944.693 & $20.672(0.171)$ & & & & \\
\hline
\end{tabular}

Table 2. Optical spectroscopy of PTF11mnb and its host-galaxy.

\begin{tabular}{cccccc}
\hline \hline Date (UT) & $\begin{array}{c}\text { JD-2 455000 } \\
\text { (days) }\end{array}$ & $\begin{array}{c}\text { Phase }^{a} \\
\text { (days) }\end{array}$ & Telescope & Instrument & $\begin{array}{c}\text { Range } \\
(\AA)\end{array}$ \\
\hline SN spectra & & & & & \\
07 Oct. 2011 & 841.98 & +35.5 & UH88 & SNIFS & 3301-9701 \\
30 Oct. 2011 & 864.78 & +57.0 & P200 & DBSP & $3002-10293$ \\
26 Nov. 2011 & 891.92 & +82.6 & Keck I & LRIS & $3001-10297$ \\
21 Dec. 2011 & 916.68 & +105.9 & P200 & DBSP & $3001-10296$ \\
27 Jan. 2012 & 953.50 & +140.7 & KPNO4m & RC Spec & 3575-8138 \\
\hline Host-galaxy spectrum & $\ldots$ & $\ldots$ & TNG & DOLORES & 3189-10352 \\
02 Sep. 2016 & $\ldots$ & $\ldots$ & & & \\
\hline
\end{tabular}

Notes. ${ }^{(a)}$ Rest frame days from explosion.

and including the main peak. In Fig. 2 we show the absolutemagnitude light curves of SN 2005bf from Folatelli et al. (2006) and Bianco et al. (2014) as solid colored lines. For SN 2005bf we assumed a distance modulus $\mu_{05 b f}=34.561 \mathrm{mag}$, redshift $z=$ 0.018913 (from NED), explosion epoch JD $=2453458$, galactic extinction $A_{B}(05 b f)=0.163 \mathrm{mag}, A_{g}(05 b f)=0.148 \mathrm{mag}$,
$A_{r}(05 b f)=0.102 \mathrm{mag}$, and $A_{i}(05 b f)=0.076 \mathrm{mag}$; the redshift is from NED ${ }^{1}$, the distance modulus is computed assuming the same cosmology as used for PTF11mnb, and the extinction is from Schlafly \& Finkbeiner 2011. Both the early $r$-band rise

\footnotetext{
1 NASA/IPAC Extragalactic Database:
}

https://ned.ipac.caltech.edu 
Table 3. Properties of PTF11mnb and its host galaxy SDSS J003413.34+024832.9.

\begin{tabular}{|c|c|c|}
\hline Parameters & Value & Ref. \\
\hline \multicolumn{3}{|l|}{ SDSS J003413.34+024832.9: } \\
\hline Position & $\begin{array}{l}\alpha_{\mathrm{J} 2000}=00^{\mathrm{h}} 34^{\mathrm{m}} 13: 34 \\
\delta_{\mathrm{J} 2000}=+02^{\circ} 48^{\prime} 32^{\prime \prime} \cdot 9\end{array}$ & Sect. 3 \\
\hline Abs. magnitude & $\begin{array}{l}M_{g}=-17.8 \mathrm{mag} \\
M_{r}=-18.0 \mathrm{mag} \\
M_{i}=-18.3 \mathrm{mag}\end{array}$ & Sect. 3 \\
\hline Redshift & $z=0.0603 \pm 0.0001$ & Sect. 3 \\
\hline Distance & $D=268.5 \mathrm{Mpc}$ & Sect. 3 \\
\hline Distance modulus & $\mu=37.14 \mathrm{mag}$ & Sect. 3 \\
\hline $\begin{array}{l}\text { Metallicity of the host } \\
\text { at the SN location (O3N2) }\end{array}$ & $\log (\mathrm{O} / \mathrm{H})+12=8.29 \pm 0.20$ & Sect. 3 \\
\hline $\begin{array}{l}\text { PTF11mnb: } \\
\text { Position }\end{array}$ & $\begin{array}{l}\alpha_{\mathrm{J} 2000}=00^{\mathrm{h}} 34^{\mathrm{m}} 13 \mathrm{~s} 25 \\
\delta_{\mathrm{J} 2000}=+02^{\circ} 48^{\prime} 31^{\prime \prime} \cdot 4\end{array}$ & Sect. 2 \\
\hline Explosion epoch & JD $2455804.341 \pm 0.516$ & Sect. 2 \\
\hline Total reddening toward SN: & $E(B-V)=0.016 \mathrm{mag}$ & Sect. 3 \\
\hline $\begin{array}{l}\text { Phase of the main peak, in } \\
\text { rest-frame days since explosion }\end{array}$ & $\begin{array}{l}t_{B}^{\max }=41.9 \mathrm{~d} \\
t_{g}^{\max }=46.3 \mathrm{~d} \\
t_{r}^{\max }=52.2 \mathrm{~d} \\
t_{i}^{\max }=48.1 \mathrm{~d}\end{array}$ & Sect. 4 \\
\hline Peak abs. magnitude & $\begin{array}{l}B_{\max }=-18.25 \mathrm{mag} \\
g_{\max }=-18.37 \mathrm{mag} \\
r_{\max }=-18.45 \mathrm{mag} \\
i_{\max }=-18.52 \mathrm{mag}\end{array}$ & Sect. 4 \\
\hline$\Delta m_{15}$ & $\begin{array}{l}\Delta B_{15}=0.48 \mathrm{mag} \\
\Delta g_{15}=0.53 \mathrm{mag} \\
\Delta r_{15}=0.16 \mathrm{mag} \\
\Delta i_{15}=0.24 \mathrm{mag}\end{array}$ & Sect. 4 \\
\hline
\end{tabular}

and the early $g$ - and $r$-band peaks are similar in phase and absolute magnitude. However, the early peak in $r$ band is more pronounced in PTF11mnb than in SN 2005bf, where the $r$-band light curve shows a change in curvature rather than a clear maximum. In $g$ band, both SNe show clear early maxima. SN 2005bf shows its main peak in $B, g$, and $r$ band slightly earlier (3$10 \mathrm{~d})$ than PTF11mnb, whereas the peak epoch is very similar in $i$ band. The Bgri peaks of SN 2005bf are only 0.2-0.3 mag fainter than those of PTF11mnb.

After the main peak, the light curves of SN 2005bf decline much faster than those of PTF11mnb. At 100 days, SN 2005bf has declined by $\sim 2.5$ mag from peak in $r$ band, whereas PTF11mnb has declined by merely $\sim 1.0 \mathrm{mag}$ in the same band.

We summarize the main light-curve properties of PTF11mnb in Table 3.

The color evolution of PTF11mnb is similar to that of SN 2005bf, as shown in Fig. 3. The early light-curve peak (see bottom panel) corresponds to an early maximum in the $g-r$ colors, whereas the main light curve peak correspond to a minimum in the $g-r$ colors. These minima are followed by a rise in the $g-r$ values in both SNe. PTF11mnb is slightly redder than SN 2005bf around the early peak, although it is bluer later on. For PTF11mnb we have late detections in $g$ and $r$, and at epochs later than 90 days $g-r$ slowly becomes bluer. The $g-r$ colors of normal SE SNe, such as those of iPTF13bvn (Fremling et al. 2016) and SN 2011dh (Ergon et al. 2014, 2015) shown in the figure, evolve differently. After the $g-r$ minimum in correspondence with the light-curve peak, the $g-r$ of these normal SE $\mathrm{SNe}$ becomes redder until $\sim 30 \mathrm{~d}$ and then slowly turns bluer.

\section{Spectra}

PTF11mnb was observed five times in spectroscopy, from $+35 \mathrm{~d}$ to $+141 \mathrm{~d}$. The spectral sequence is shown in Fig. 4. The spectra will be available via WISeREP (Yaron \& Gal-Yam 2012).

We did not remove the narrow emission lines due to the host galaxy from the SN spectra. These are well visible in Fig. 4. We can observe narrow $\mathrm{H} \alpha, \mathrm{H} \beta$, [O III] $\lambda \lambda 4959,5007$, [O II] $\lambda 3727$, and S II $\lambda \lambda 6717,6731$ emission lines. Aside from the narrow emission lines, the host-galaxy continuum emission (which peaks in the blue, see Fig. 1) could not be completely removed and this emission is likely contaminating the continuum of the spectra, affecting their shape in particular at late epochs.

The spectra of PTF11mnb are similar to other SN Ic spectra, as illustrated in Fig. 5 (top panel). Some of the best SN Ic spectral fits obtained with SNID $^{2}$ (Blondin \& Tonry 2007) are shown for comparison, and they clearly match the features of PTF11mnb. On the other hand, the spectra of PTF11mnb are remarkably different from those of SN 2005bf (see Fig. 5, top panel), which show increasingly stronger He lines. PTF11mnb never became a SN Ib.

The spectra of PTF11mnb show the characteristic lines of a SN Ic dominating over the continuum from the second spectral epoch. We provide line identifications using the spectrum with best signal $(+83$ d) shown in Fig. 5 (bottom panel) and compare these line identifications with those of SE SN spectra from the literature (Taubenberger et al. 2006; Parrent et al. 2007; Hunter et al. 2009). Neither $\mathrm{H}$ nor He lines are detected. The spectrum is dominated by Ca II, O I, and Fe II lines. Ca II and O I characterize the red part of the spectrum with P-Cygni profiles. Lines from $\mathrm{Na}$ I D, Mg II, and C I are also visible, as observed in other SE SNe (see, e.g., Elmhamdi et al. 2006). We can also see lines due to Sc II and Ba II, which are not very common in SE SNe, but appear in SNe II (e.g., Taddia et al. 2016b).

In the last two spectra, [OI] $\lambda \lambda 6300,6364$ is observed in emission. In Fig. 6 we observe that the emission lines [OI] $\lambda \lambda 6300,6364$ detected in the spectra of PTF11mnb at $>100 \mathrm{~d}$ are blueshifted. After continuum subtraction, the shape of these two lines can be reproduced by the sum of two Gaussians with the same FWHM, which have peaks at $64 \AA$ from each other and have a relative flux ratio of 1:3 (see Milisavljevic et al. 2010, for a similar analysis). This is also the case for the [OI] $\lambda \lambda 6300,6364$ lines in the nebular spectrum of SN 2005bf from Modjaz et al. (2008), which we show in Fig. 6. The individual Gaussian components are shown by dashed curves and the total best fit is shown with solid curves for both SNe. The vertical dashed lines (black for PTF11mnb, blue for SN 2005bf) indicate that the blueshift is larger for SN 2005bf $\left(-2260 \mathrm{~km} \mathrm{~s}^{-1}\right)$ than in PTF11mnb $\left(-1640 \mathrm{~km} \mathrm{~s}^{-1}\right)$. Apart from the $[\mathrm{OI}]$ emission lines, the spectra of PTF11mnb are not fully nebular even at these late epochs.

From the first four spectra we measured the P-Cygni absorption velocities of some of the most prominent lines. We report them in Fig. 7. Ca II shows a constant velocity of about

\footnotetext{
2 Supernova identification:

https://people.lam.fr/blondin.stephane/software/snid/
} 


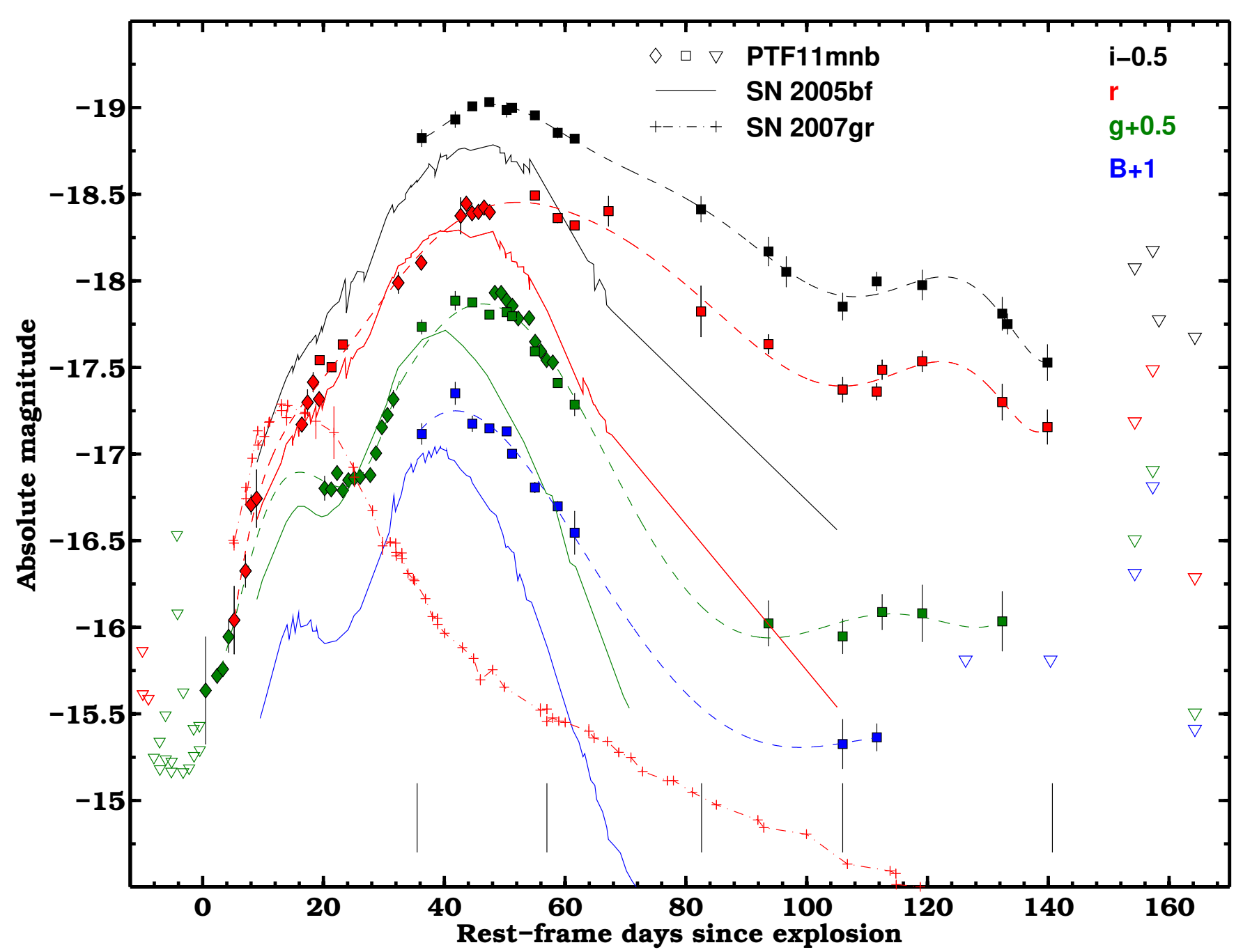

Fig. 2. Optical (Bgri) light curves of supernova PTF11mnb (colored symbols) compared to those of SN 2005bf (solid lines; Folatelli et al. 2006; Bianco et al. 2014). The light curves of both events have been shifted in the same way, as indicated in the top-right corner. The five spectral epochs for PTF11mnb are indicated with vertical black segments at the bottom of the plot. The light curves of PTF11mnb were fit with loworder polynomials (dashed lines) to characterize their shape. Limiting magnitudes are indicated with empty triangles. P48 data are indicated with diamonds, P60 data by squares. Until and including the main peak, the light curves of SN 2005bf and PTF11mnb resemble each other, whereas after the main peak, PTF11mnb, shows a much slower decline rate. We also compare the $r$-band light curves of PTF11 mnb and SN $2005 \mathrm{bf}$ to the $R$-band light curve of the normal SN Ic $2007 \mathrm{gr}$ (Hunter et al. 2009). While the first two SNe show the main peak at $\sim 52 / 42 \mathrm{~d}$ and at $-18.5 /-18.3 \mathrm{mag}$, SN $2007 \mathrm{gr}$ peaks at a similar phase (at $\sim 16 \mathrm{~d})$ and magnitude $(\sim-17.2 \mathrm{mag}$ ) as the early peak/bump of the other two events.

$6000 \mathrm{~km} \mathrm{~s}^{-1}$, O I $\lambda 7774$ is detected at about the same velocity in the first spectrum and later on at $\sim 5000 \mathrm{~km} \mathrm{~s}^{-1}$. A similar velocity is shown by $\mathrm{Na}$ I D and Fe II $\lambda 5169$. The expansion velocities of PTF11mnb as measured from the Fe II $\lambda 5169$ line are slower than those of normal SNe Ic (Modjaz et al. 2016) at similar epochs $\left(\sim 4500 \mathrm{~km} \mathrm{~s}^{-1}\right.$ instead of $\left.\sim 7000 \mathrm{~km} \mathrm{~s}^{-1}\right)$. SN $2005 \mathrm{bf}$ display higher velocities than PTF11 mnb when we compare the Fe II lines, and PTF11mnb does not have spectra taken early enough to check if there were fast velocity components in Fe II and Ca II as observed in SN 2005bf (see Folatelli et al. 2006).

\section{Modeling}

\subsection{Bolometric properties}

In order to build a quasi-bolometric light curve of PTF11mnb, we made use of its broadband photometry. We interpolated the gri light curves to the epochs of the P60 $r$ band. Then we converted the interpolated magnitudes (corrected for the extinction) into fluxes at the effective wavelength of the corresponding filters (Fukugita et al. 1996). The resulting spectral energy distributions (SEDs) are integrated and multiplied by $4 \pi D^{2}$, where $D$ is the luminosity distance to the supernova. By doing this, we obtained $L_{\text {gri }}$, which is indicated with empty red diamonds in Fig. 8 (top panel). To account for the emission at bluer and redder wavelengths, we fit the gri SEDs with a blackbody (BB) function, which we then integrated to derive $L_{\mathrm{BB}}$ (empty black diamonds in Fig. 8, top panel). As the BB fit tends to overestimate the flux in the blue, where metal lines typically absorb a significant fraction of flux, we resorted to use the bolometric corrections by Lyman et al. (2014) along with $g$ and $r$ band to derive the final bolometric light curve $\left(L_{\mathrm{Bol}}\right.$; red diamonds in the top panel of Fig. 8). By using only $g$ and $r$ along with the bolometric corrections, we also produced the rising part of the bolometric light curve, since only those two filters were used at that phase. Among other events, SN 2005bf was also used by Lyman et al. (2014) to construct the bolometric corrections for SE SNe that we use here for PTF11mnb. Our bolometric 


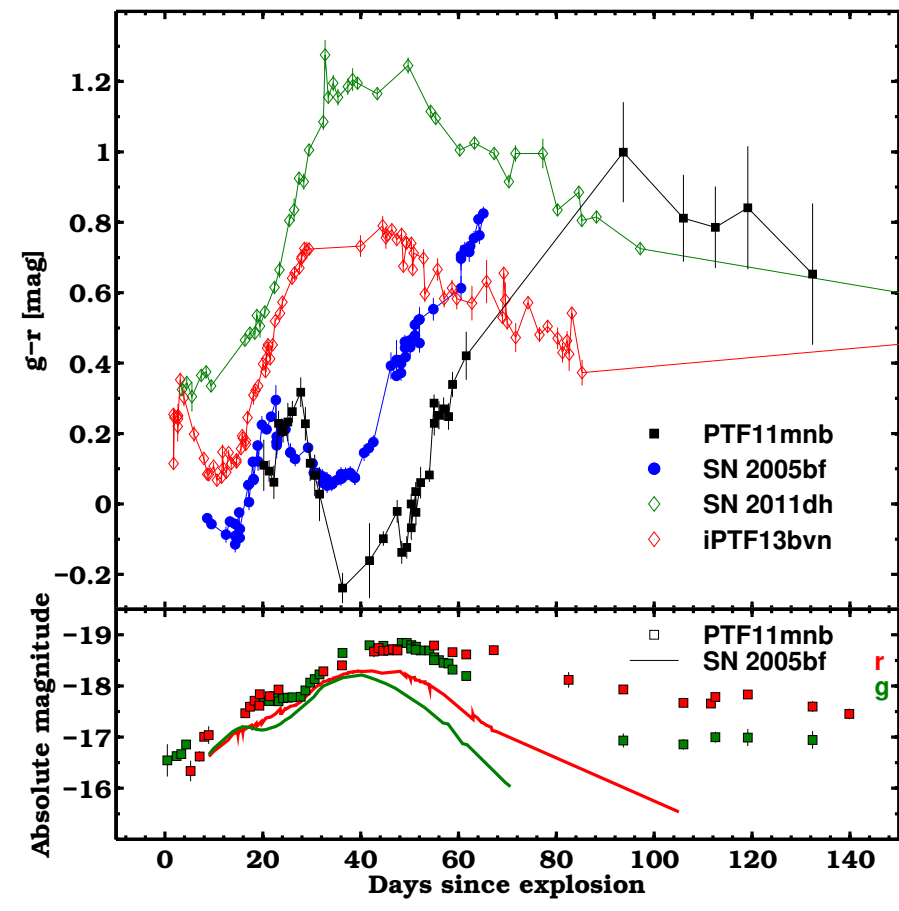

Fig. 3. Top panel: color comparison of $g-r$ between PTF $11 \mathrm{mnb}$, SN 2005bf, SN Ib iPTF13bvn (Fremling et al. 2016), and SN IIb 2011dh (Ergon et al. 2014, 2015). The first two SNe reach a red peak in the color, which coincides with the early peak in their light curves (see bottom panel). Then their $g-r$ becomes bluer reaching a minimum when the main peak of the light curves occurs. Thereafter, they rapidly evolve to redder colors. PTF11mnb also shows a shallow trend to the blue after $\sim 100 \mathrm{~d}$, when the light curves are flatter (see bottom panel) and when SN 2005bf was not observed. iPTf13bvn and SN 2011dh evolve very differently, reaching a $g-r$ minimum at peak magnitude, followed by a linear trend to the red and a final flatter color after $\sim 30 \mathrm{~d}$. Bottom panel: absolute $g$ - and $r$-band light curves of PTF11mnb and SN 2005bf, plotted as a reference for the various phases of the color evolution.

light curve is compared to that of SN 2005bf from Folatelli et al. (2006) in the top panel of Fig. 8. The two light curves are almost identical until and including peak, thereafter SN 2005bf drops much faster, whereas PTF11 mnb seems to follow the decay rate of ${ }^{56} \mathrm{Co}$ (shown by a black dashed line) with the slope of $M_{\mathrm{Bol}}$ at epochs later than $70 \mathrm{~d}$ that is $0.011 \pm 0.03 \mathrm{mag} \mathrm{d}^{-1}$.

As a byproduct of the BB fit to the SEDs, we also obtained the temperature and velocity evolution (derived from the $\mathrm{BB}$ radius divided by the time since explosion) of PTF11mnb, which is reported in the central and bottom panels of Fig. 8 (black diamonds). This evolution is rather similar to that of SN 2005bf (Folatelli et al. 2006), which is shown by a solid black line. A maximum in the temperature is reached around the epoch of the second light-curve peak in both $\mathrm{SNe}$, as already suggested by the color evolution (Fig. 3). The velocity has a peak around $\sim 60 \mathrm{~d}$ in both $\mathrm{SNe}$.

\subsection{Double-peaked ${ }^{56} \mathrm{Ni}$ distribution scenario}

The bolometric light curve of PTF11mnb and its velocity and temperature evolution can be reproduced by a model similar to that suggested for SN 2005bf by Tominaga et al. (2005) and Folatelli et al. (2006). This considers the explosion of a massive star characterized by a double-peaked distribution of ${ }^{56} \mathrm{Ni}$. A relatively small fraction of ${ }^{56} \mathrm{Ni}$ in the outer layers makes it possible to power the early peak. A larger fraction of ${ }^{56} \mathrm{Ni}$ situated deeper in the ejecta allows us to reproduce the main peak. In the case of SN 2005bf, the post main-peak decline was fast and required an artificially reduced gamma-ray trapping in this scenario. The late-time photometry of SN 2005bf could not be reproduced with a radioactive model, so a magnetar was invoked by Maeda et al. (2007). Contrary to SN 2005bf, we do not need to reduce the gamma-ray opacity artificially by a factor of 10 to match the post main-peak bolometric light curve. The slow decline of PTF $11 \mathrm{mnb}$ is instead fully consistent with the decay rate of ${ }^{56} \mathrm{Co}$ (see black dashed line in Fig. 8).

As the first step to model PTF11mnb, we produced a massive (final mass 9.5 $M_{\odot}$ ) pre-SN star with the Modules for Experiments in Stellar Astrophysics (MESA; Paxton et al. 2011), similar to what was used by Folatelli et al. (2006) to model SN 2005bf. We started from a star with initial mass $M_{\text {ZAMS }}=$ $85 M_{\odot}$ and a slightly subsolar metallicity $(Z=0.01)$, which is consistent with what we inferred for the location of PTF11mnb (see Sect. 3). We set the star rotation velocity to $350 \mathrm{~km} \mathrm{~s}^{-1}$. The rotation and initial mass were adjusted to reproduce the desired final mass and at the same time to strip the entire hydrogen envelope and the helium envelope. The final progenitor star model contains merely $0.6 M_{\odot}$ of $\mathrm{He}$ in the outer part of the ejecta, which may be consistent with a SN Ic progenitor (see, e.g., the progenitor star model 5p11 in Dessart et al. 2015).

In Fig. 9 we report the evolution of this star in the Hertzprung-Russell (HR) diagram, until collapse. In the final stage of its life this star sits in the Wolf-Rayet (WR) part of the HR diagram (see blue diamond) with high luminosity ( $10^{5.5}$ solar luminosity), high temperature (almost $10^{5.36} \mathrm{~K}$ ), and a compact radius $\left(0.35 R_{\odot}\right)$.

We exploded this star with the SuperNova Explosion Code (SNEC; Morozova et al. 2015). For an explosion energy of $E=$ $2.2 \times 10^{51} \mathrm{erg}$, a remnant mass of $1.7 M_{\odot}$ (i.e., an ejecta mass of $7.8 M_{\odot}$ ), and using the double-peaked ${ }^{56} \mathrm{Ni}$ distribution plotted in Fig. 10, which was slightly modified from that of Folatelli et al. (2006), we reproduced the bolometric light curve of PTF11mnb rather accurately (see the thick red dashed lines in Fig. 8). The total ${ }^{56} \mathrm{Ni}$ mass of this model is $0.59 M_{\odot}$. It is necessary to use a double-peaked ${ }^{56} \mathrm{Ni}$ distribution to reproduce both the early and main peak. We modified the SNEC code to include the nonuniform ${ }^{56} \mathrm{Ni}$ distribution shown in Fig. 10.

The model is also able to somewhat reproduce the photospheric-velocity profile as measured from the Fe II $\lambda 5169$ line in the spectrum and the temperature evolution. We corrected the SNEC temperature profile by the ratio between the photospheric temperature and VI color temperature for $\mathrm{SNe} \mathrm{Ibc}$, presented by Dessart et al. (2015) in their table A3, to compare directly the photospheric temperature from the SNEC model and BB temperature from the fit of the gri SEDs.

An important assumption in the hydrodynamical model is that related to the opacity floor. Following Morozova et al. (2015), we adopted a linear scaling of the opacity floor with metallicity. In particular, we started our model investigation with $0.01 \mathrm{~cm}^{2} \mathrm{~g}^{-1}$ at $Z=0.02$ (in the envelope) and $0.025 \mathrm{~cm}^{2} \mathrm{~g}^{-1}$ in the core, where $Z=1$. The opacity floor value in the envelope is the same as adopted for SNe II and SNe IIb in Bersten et al. (2011) and Ergon et al. (2015), respectively. The opacity floor in the core is the same as adopted for hydrogen-poor, and helium-rich SNe (see Ergon et al. 2015). This value was calibrated with a sophisticated radiative transfer code, STELLA (Blinnikov \& Bartunov 1993). However, the optimal value of the opacity floor for SNe Ic, i.e., for helium-poor SNe, has not been presented in the literature yet. Therefore, we started our model investigation by assuming that the opacity floor in the core is 


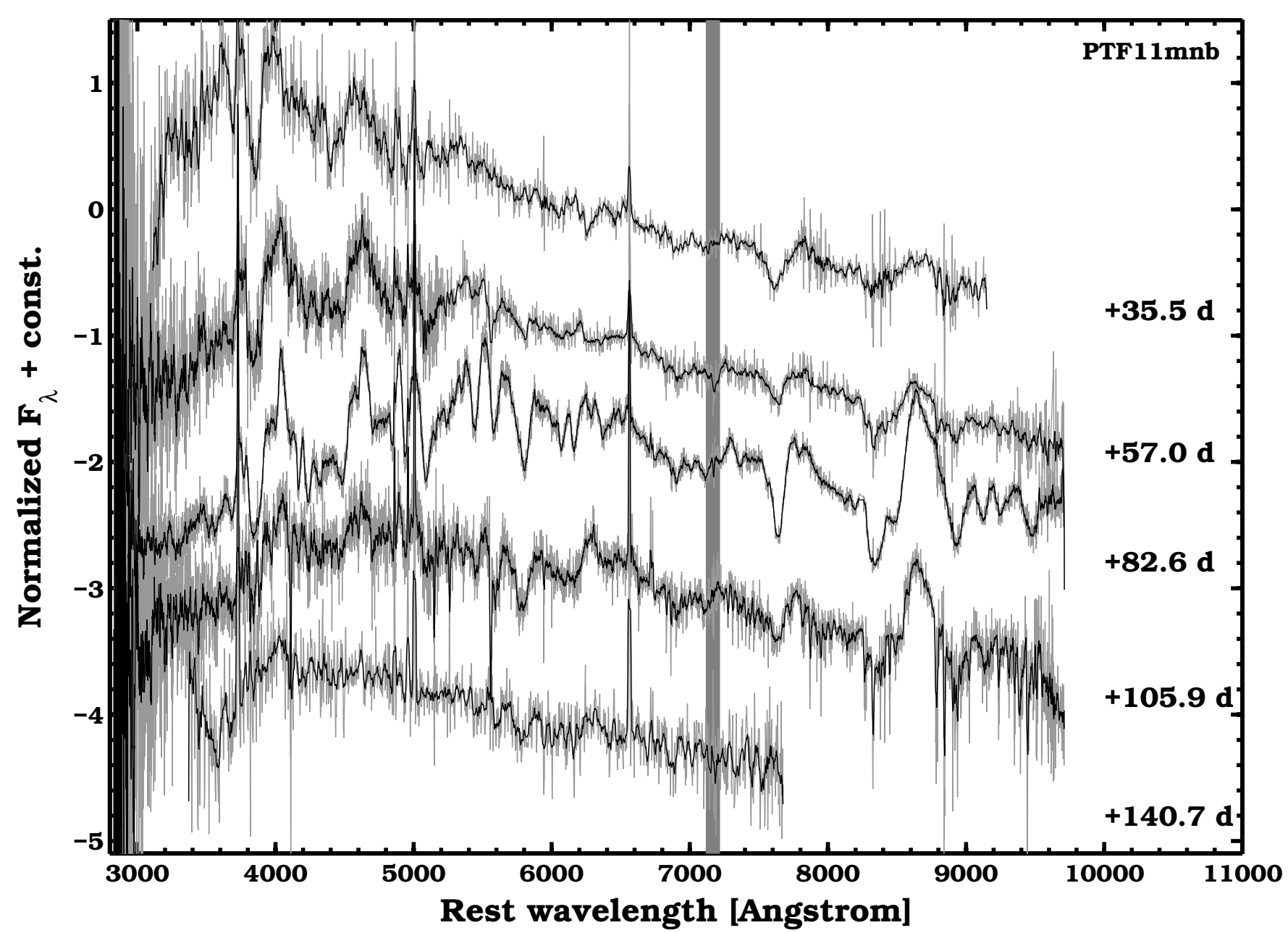

Fig. 4. Spectral sequence of PTF11mnb. Phases in rest-frame days since explosion are reported next to each spectrum. A gray area masks the strongest telluric feature at $7600 \AA$.

similar for SNe IIb and SNe Ic, which are both hydrogen poor. Our model with the core opacity floor set to $0.025 \mathrm{~cm}^{2} \mathrm{~g}^{-1}$ succeeds in reproducing the velocity profile and light curve. However, around peak it shows a narrow feature in the light curve that is not observed in our SN. By slightly modifying the opacity floor in the core, i.e., adopting $0.04 \mathrm{~cm}^{2} \mathrm{~g}^{-1}$, we were able to smooth the narrow feature around the peak of the light-curve model while preserving the good match to the velocity profile from the Fe II $\lambda 5169$ line. For higher values of core opacity floor, the light-curve match is still good until $0.07 \mathrm{~cm}^{2} \mathrm{~g}^{-1}$ and it is acceptable to adopt $0.1 \mathrm{~cm}^{2} \mathrm{~g}^{-1}$. However, the velocities for the models with higher values of core opacity floor vare above those observed in our SN. For even higher values of opacity floor, in particular if we use the core opacity floor for hydrogen-rich SNe II presented by Bersten et al. (2011) $\left(0.24 \mathrm{~cm}^{2} \mathrm{~g}^{-1}\right)$ and used by Morozova et al. (2015), the light-curve model instead shows an extremely long rise time, which is not compatible with the observed light curve (the other parameters being the same).

\subsection{Magnetar scenario}

For SN 2005bf, Maeda et al. (2007) proposed a spinning magnetar as the main powering source of the light curve based on the fast decline after the main peak and on late-time photometry. These properties were difficult to reconcile with a scenario in which the radioactive decay was the powering mechanism.
If we fit the bolometric light curve after $+30 \mathrm{~d}$ with a magnetar model (Kasen \& Bildsten 2010), we obtain ejecta mass $M_{e j}=3 M_{\odot}$, kinetic energy $E_{K}=0.6 \times 10^{51} \mathrm{erg}$, magnetic flux density $B=4.3 \times 10^{14} G$, rotation period $P=14.2 \mathrm{~ms}$. Here we have assumed an expansion velocity at peak of $5600 \mathrm{~km} \mathrm{~s}^{-1}$ from the Fe II velocity, and opacity $\kappa=0.2 \mathrm{~cm}^{2} \mathrm{~g}^{-1}$. This simple model cannot reproduce the early peak, and therefore we fit only the later epochs; however, including all the epochs provides almost identical parameters. To reproduce the early peak in the magnetar scenario as well, we consider the early light curve as possibly produced by a magnetar-induced shock breakout (SBO), as described by Kasen et al. (2016). Using the above derived parameters from the fit of the main peak with the magnetar model, we can derive the time of the SBO peak and its luminosity (Kasen et al. 2016, their Eqs. (26) and (27)). Both these values are off from what we actually observe in PTF11mnb; we obtained a peak epoch for the SBO of 83 days, with luminosity $3.5 \times 10^{43} \mathrm{erg}$. Therefore, we do not favor a magnetar as the energy source for the early peak.

Since a magnetar-induced SBO model does not fit the early light curve of PTF $11 \mathrm{mnb}$, whereas the early peak was nicely fit by a ${ }^{56} \mathrm{Ni}$ decay model (see Sect. 6.2), here we also consider a hybrid model in which the first peak is due to a regular SN explosion powered by radioactivity, while the main peak is powered by a magnetar. It is indeed possible to fit the bolometric light curve with a simple Arnett model (Arnett 1982) 

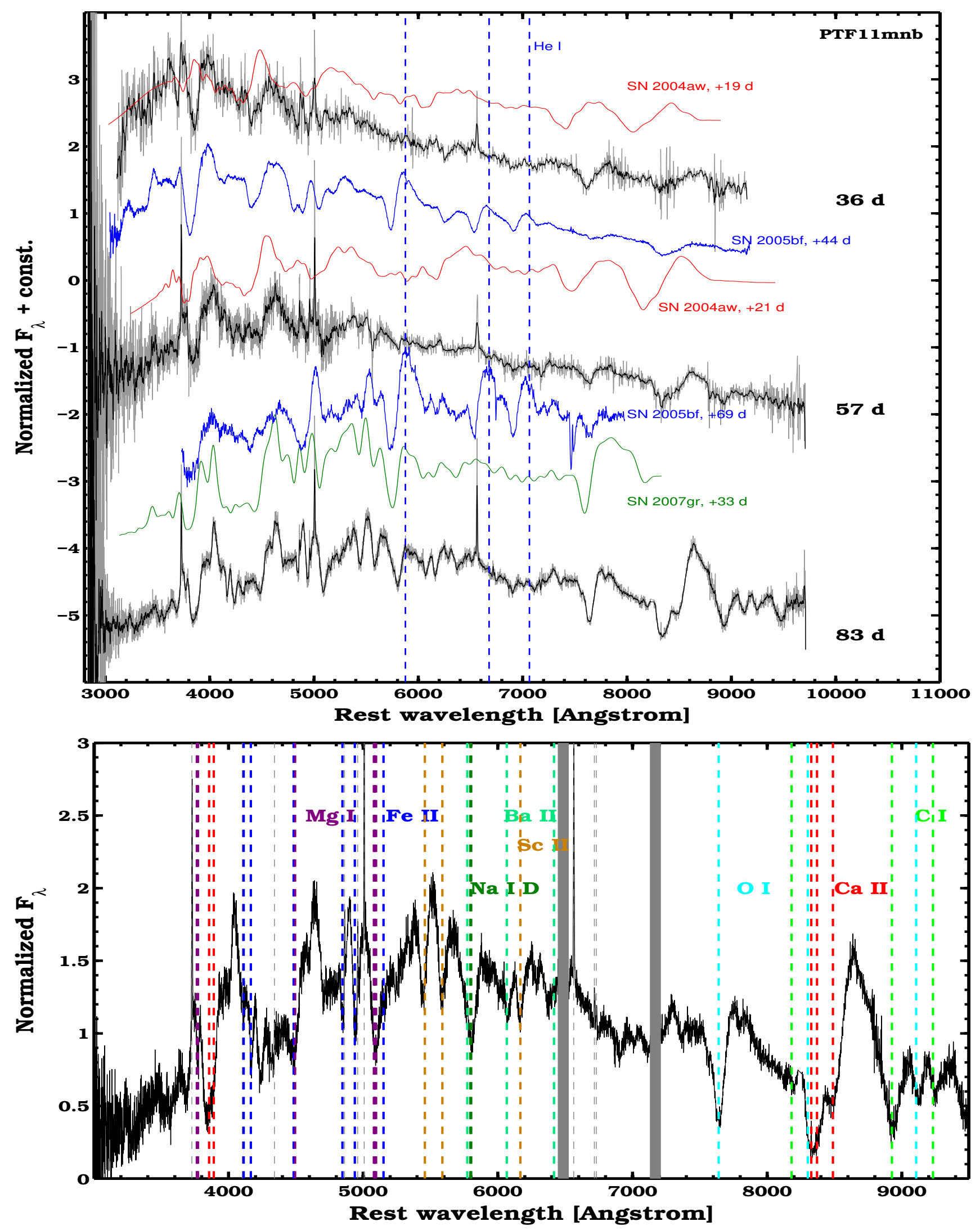

Fig. 5. Top panel: spectral comparison of PTF11mnb to other SNe Ic (from SNID templates, Blondin \& Tonry 2007; Liu \& Modjaz 2014) and SN 2005bf (Folatelli et al. 2006). Phases from explosion in rest-frame days are reported next to each spectrum. SN 2005bf is the only SN showing strong He lines, which are not present in PTF11mnb. Bottom panel: line identification on the best PTF11mnb spectrum. Typical SN Ic features dominate the spectrum, with ions such as Ca II, O I, Fe II, Mg I, C I, and Na I. Lines from ions such as Sc II and Ba II are also visible. Dashed lines of the same color indicate the same ion, which is labeled in the plot with the same color. Gray areas represent the most prominent telluric features. 


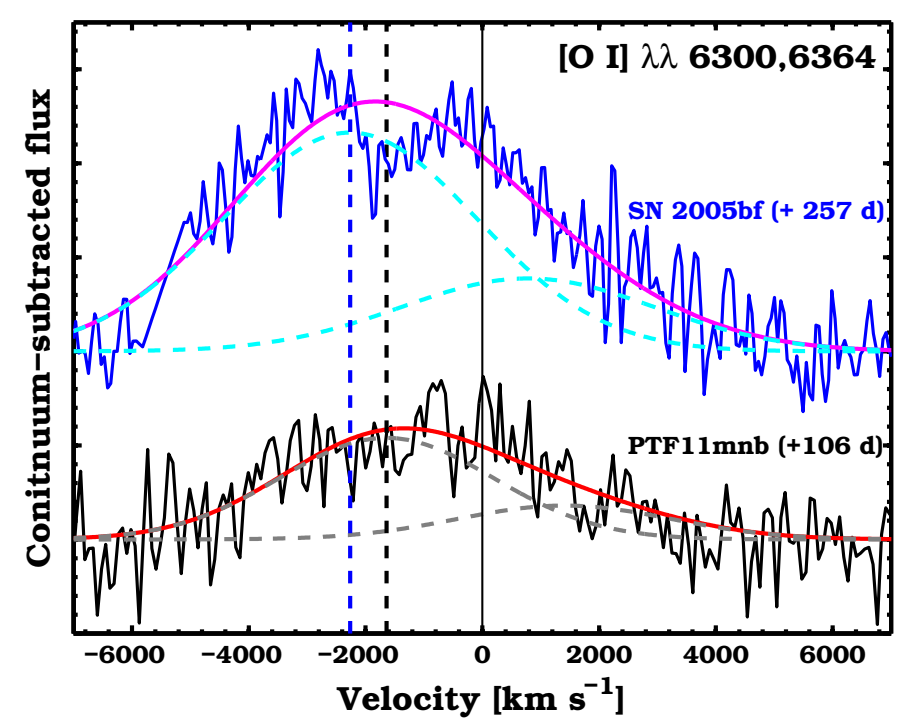

Fig. 6. [O I] $\lambda \lambda 6300,6364$ lines from the fourth spectrum of PTF11mnb $(+106 \mathrm{~d}$, black line) as compared to the same lines in a late spectrum of SN 2005bf (from Modjaz et al. 2008, blue line). The [O I] $\lambda \lambda 6300,6364$ lines are shown in velocity space and continuum subtracted, after fitting the continuum with a low order polynomial. The lines are fitted with the sum (solid curves) of two Gaussians (shown as dashed curved), with the same FWHM, relative flux ratio of 1:3, and peaks separated by $64 \AA$. The [OI] $\lambda 6300$ line peaks are indicated with thick dashed lines, at $-1640 \mathrm{~km} \mathrm{~s}^{-1}$ for PTF11mnb and $-2260 \mathrm{~km} \mathrm{~s}^{-1}$ for SN 2005bf. The zero velocity of the line (at $6300 \AA$ ) is indicated with a solid, vertical black line.

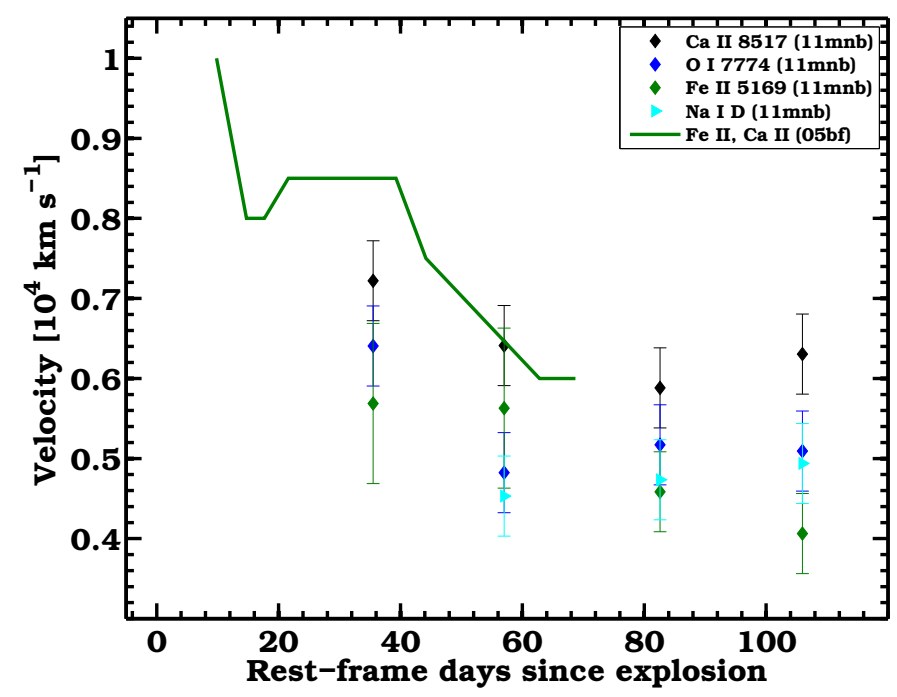

Fig. 7. Expansion velocities of PTF11mnb as measured from the absorption minima of some spectral lines showing P-Cygni profiles. We also report the Fe II and Ca II velocities of SN 2005bf for comparison. SN 2005bf also shows high and low velocity components for these lines, as shown by Folatelli et al. (2006).

representing the radioactive component, plus a magnetar model (Kasen \& Bildsten 2010) that injects energy in the ejecta starting a few days after explosion. This is illustrated in Fig. 11, where the Arnett model is plotted as a dotted blue line and the magnetar model by a dashed black line. The sum of the two models, shown as a dashed red line, fit the bolometric light curve well. The magnetar injection of energy would have to start after 18 days from

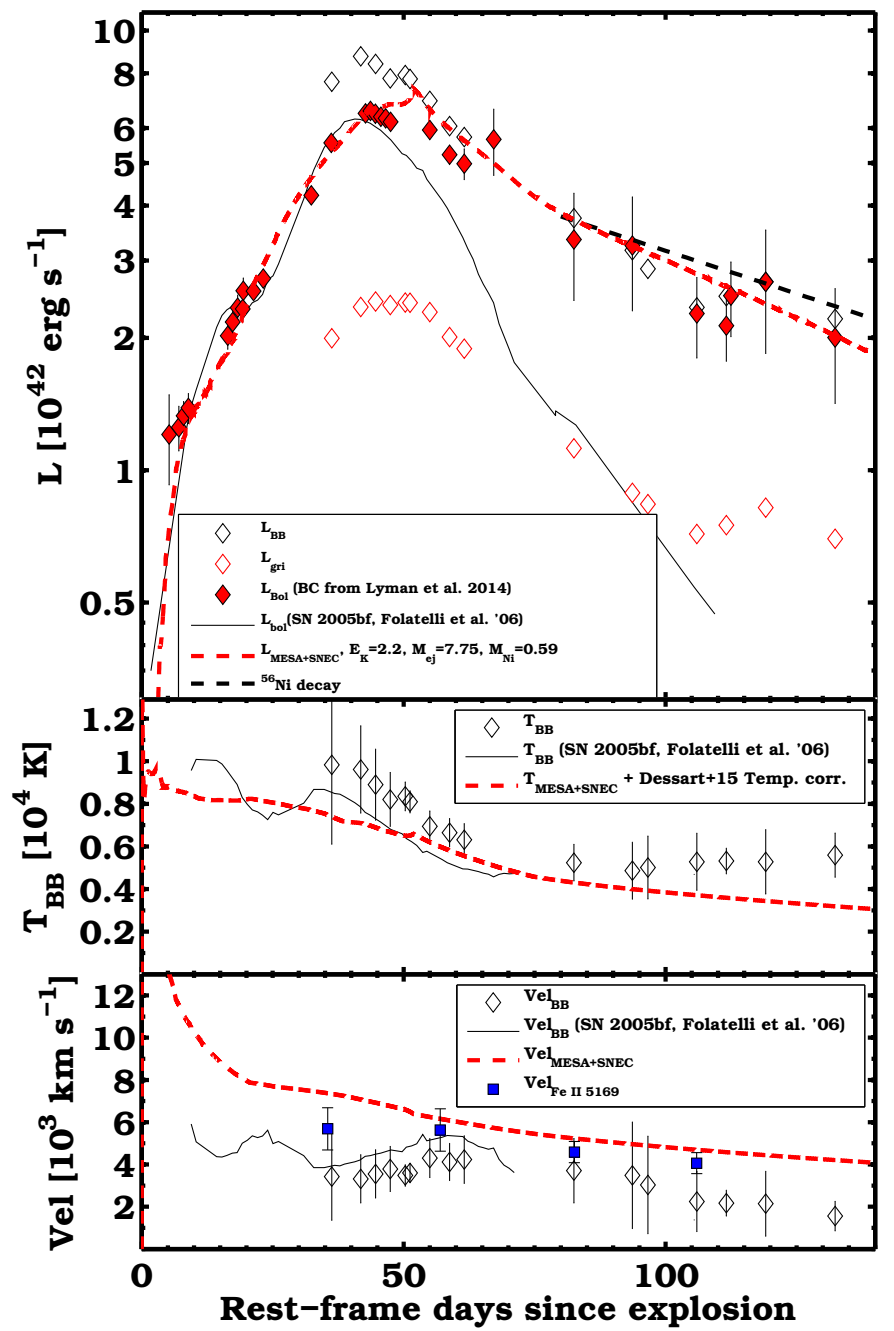

Fig. 8. Top panel: bolometric light curve of PTF11mnb. The luminosity from the integrated gri SEDs, from the BB fit to the SED, and from $g$ and $r$ along with the bolometric corrections by Lyman et al. (2014) are indicated with empty red diamonds, empty black diamonds, and red diamonds, respectively. The light curve of SN $2005 \mathrm{bf}$ from Folatelli et al. (2006) is plotted as a black solid line. The luminosity from the ${ }^{56} \mathrm{Co}$ decay $\left(M\left[{ }^{56} \mathrm{Ni}\right]=0.59 M_{\odot}\right)$ is indicated with a black dashed line. The preferred hydrodynamical model is shown as a red dashed line. Central panel: BB temperature evolution of PTF11mnb (black diamonds) is compared to that of SN 2005bf from Folatelli et al. (2006) and that of the preferred hydrodynamical model (red dashed line). Bottom panel: BB velocity evolution of PTF11 mnb (black diamonds) is compared to that of SN 2005bf from Folatelli et al. (2006), to the photospheric velocity of the preferred hydrodynamical model (red dashed line), and to the Fe II P-Cygni absorption velocity of PTF11 mnb (blue squares).

the SN explosion, otherwise it is not possible to reproduce both peaks simultaneously.

In such a scenario, the $\mathrm{SN}$ explosion is characterized by $M_{e j}=1 M_{\odot}, E_{K}=0.2 \times 10^{51} \mathrm{erg}, M^{56} \mathrm{Ni}=0.11 M_{\odot}$, and the magnetar by $B=5.0 \times 10^{14} G$ and $P=18.1 \mathrm{~ms}$. The magnetar parameters in the hybrid model are different from those of the magnetar-only model previously described. Despite the nice fit of this hybrid model to the bolometric light curve, we still favor a progenitor scenario with the explosion of a massive star and a double-peaked ${ }^{56} \mathrm{Ni}$ distribution because such a scenario requires a single source of energy and fewer parameters and, in particular, it naturally fits the post main-peak light curve. 


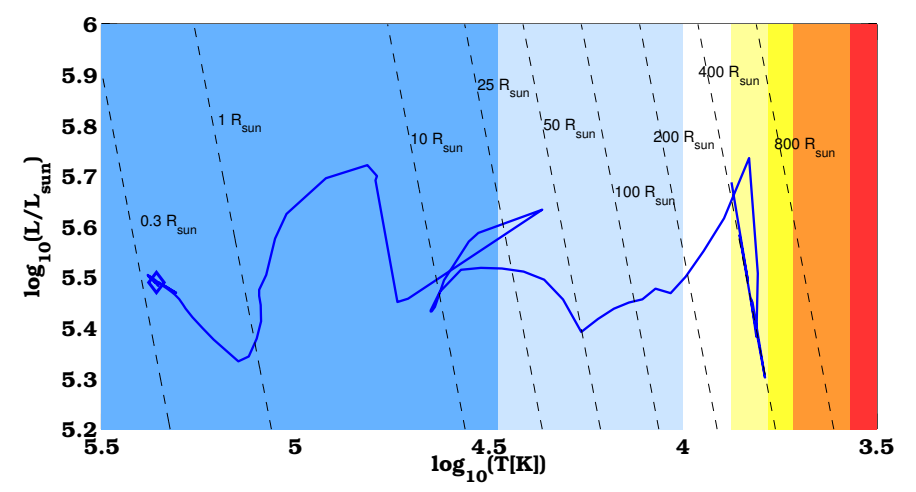

Fig. 9. HR-diagram evolution of the star produced with MESA and used to model the bolometric light curve of PTF11mnb with SNEC.

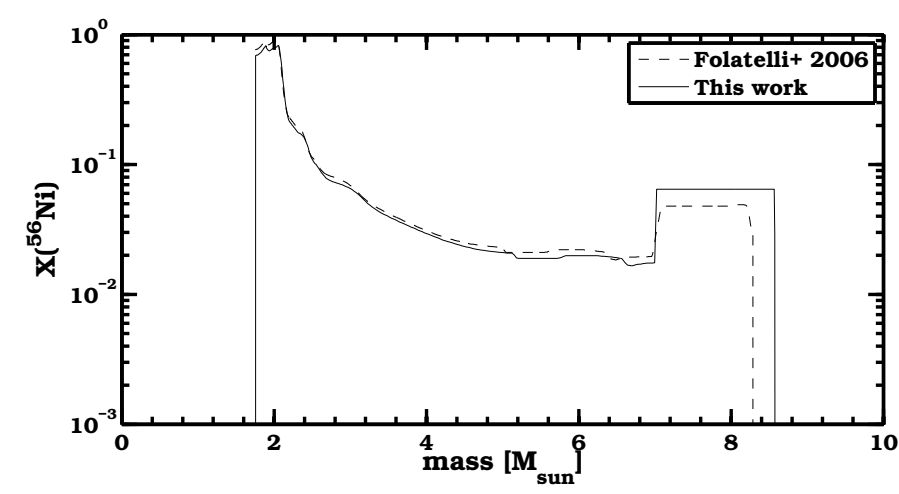

Fig. 10. ${ }^{56} \mathrm{Ni}$ distribution used for the best hydrodynamical model of PTF11mnb, as compared to that used for SN 2005bf by Folatelli et al. (2006). Both are double-peaked to reproduce both the early and the main peak in the bolometric light curve.

\subsection{Early light-curve modeling}

The early $g$-band light curve of PTF11mnb appears rather flat in the first three epochs, which occur merely $\lesssim 4 \mathrm{~d}$ after the last non-detection. To reproduce this early emission, we adopted the model by Piro \& Nakar (2013) for the shock breakout cooling tail, assuming $\kappa=0.2 \mathrm{~cm}^{2} \mathrm{~g}^{-1}$ and kinetic energy and ejecta mass from the hydrodynamical fit to the bolometric light curve. We solved for the explosion epoch and for the progenitor radius, obtaining a radius of $\sim 90-105 R_{\odot}$ and an explosion epoch at $\sim-3 \mathrm{~d}$ since the average between discovery and last nondetection. This explosion epoch is consistent with the depth of the pre-discovery limits, as shown in Fig. 12. Explosions epochs earlier than that would not be compatible with the pre-detection limits.

The best fit for the first three epochs of $g$ band is shown in the top panel of Fig. 12 (green-dotted line). The corresponding $r$-band light curve model (red-dotted line) is also shown, however the first epoch of $r$ band occurs too late to better constrain the radius and explosion epoch. In the bottom left panel of Fig. 12 we show the reduced chi-square as a function of the progenitor radius andexplosion epoch, from which we estimated the range of the best fit parameters. The best fit is shown by a white cross and corresponds to a progenitor radius of $95 R_{\odot}$. This is rather large compared to that of a regular WR star, which is about two orders of magnitude more compact. The progenitor star might have exploded while its radius was more extended than that of a WR star. This is also why the hydrodynamical

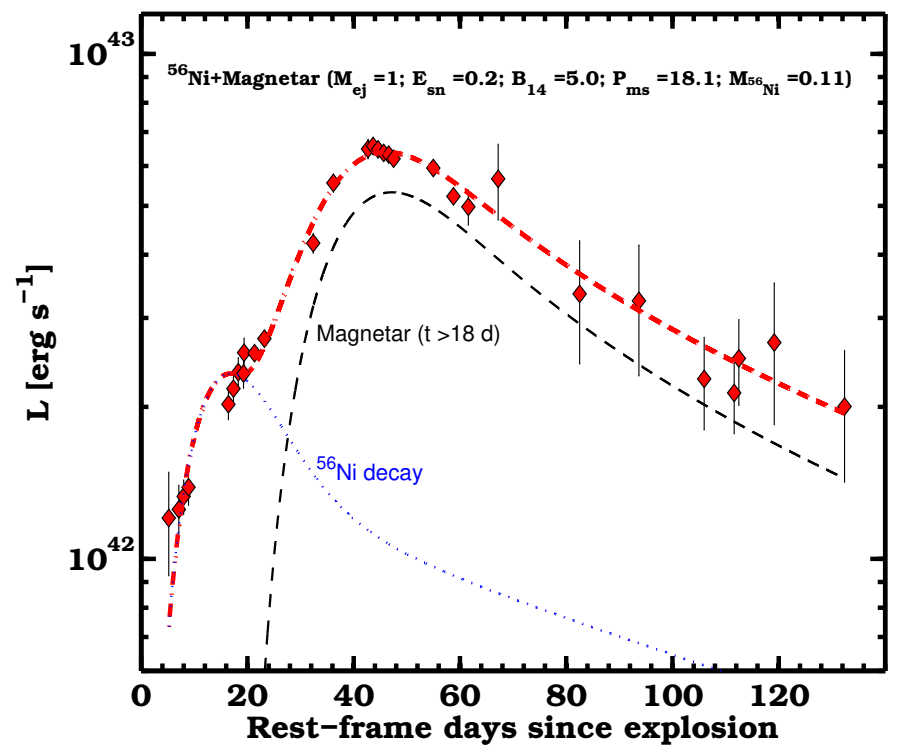

Fig. 11. Hybrid bolometric light-curve model (red dashed line) of PTF11 mnb. The first peak is fit by the radioactive decay of ${ }^{56} \mathrm{Ni}$ and represented by an Arnett model (blue dotted line). The main peak is powered by a magnetar model (dashed black line) from Kasen \& Bildsten (2010), which kicks in 18 days after explosion. The best fit parameters are reported at the top of the plot.

model could not reproduce this early emission, as we exploded a compact $\left(0.35 R_{\odot}\right)$ star.

However, another possibility is that an extended envelope of low mass surrounded the WR progenitor star at the moment of the explosion. This is how we interpreted the early emission from the explosion of the massive SN Ic progenitor of iPTF15dtg (Taddia et al. 2016a). If we fit the model by Piro (2015) that describes the early emission from a star with an extended envelope, again assuming the same opacity, ejecta mass, and explosion energy as in the previous model, we can solve for the radius and mass of the outer envelope (and for the explosion epoch). We obtained an extended envelope of mass $\sim 0.11 M_{\odot}$ and of radius $\sim 35 R_{\odot}$. These parameters are rather well constrained by the fit (see Fig 12, bottom right panel). Here the best explosion epoch was fixed to occur merely one day before the average between discovery and last non-detection to optimize the fit to the first $g$-band point. The best fit of the extended-envelope model to the $g$-band light curve is shown in Fig. 12 as a dashed green line. The corresponding $r$-band model is shown with a red dashed line and is not in contradiction with the first $r$-band observation, which however occurs too late to be used for further constraints.

Discriminating between the two models is not easy as we lack multi-band coverage at that phase. With additional $r$-band observations, we could have constrained the temperature and observed if $g-r$ was more similar to what is predicted by the extended-envelope model (Piro 2015) or by the shock breakout cooling model (Piro \& Nakar 2013). We could do that for iPTF15dtg (Taddia et al. 2016a), where we had multi-band coverage. We favor the extended-envelope model as the fit is slightly better and because PTF11mnb is somewhat similar to iPTF15dtg and SN $2011 \mathrm{bm}$, where we inferred the presence of an extended envelope.

There could also be the possibility that the early emission is due to the afterglow of a long-duration GRB. We performed a search for a possible GRB association via the interplanetary network (IPN), as in Taddia et al. (2016a). In a longer interval 

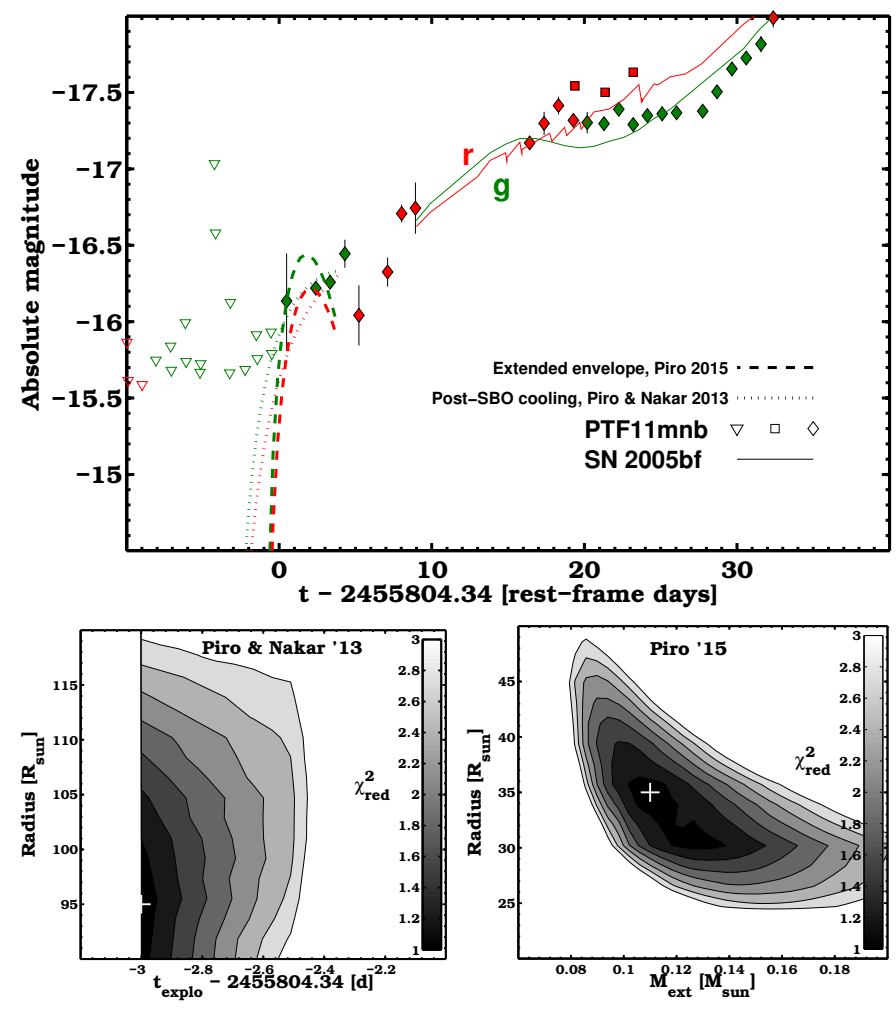

Fig. 12. Top panel: early $g$ - and $r$-band light curves of PTF $11 \mathrm{mnb}$ as compared to those of SN 2005bf. Squares and diamonds indicate P60 and P48 data, respectively. Triangles indicate upper limits. PTF11mnb was discovered earlier and does not show a regular rising light curve in the first 3 epochs of $g$-band data. We fit these epochs with a shock breakout (SBO) cooling tail model from Piro \& Nakar (2013) and with an extended envelope model from Piro (2015). The best fit of the latter model reveals the likely presence of a relatively extended envelope around the progenitor star $\left(\sim 35 R_{\odot}\right)$. Bottom panels: reduced chi square for the two $g$-band light curve fits; Piro \& Nakar (2013) fits are shown on the left and Piro (2015) fits are shown. When fitting the model by Piro \& Nakar (2013) we solved for the explosion epoch andprogenitor radius. When fitting the model provided by Piro (2015) we solved for the extended envelope radius and mass; the explosion epoch was fixed to $-1 \mathrm{~d}$ since the previously assumed value.

from seven days before discovery to discovery, seven sources are found in the catalog. However, when we cross-checked with the integral archive for the source position, none of these sources turned out to be compatible with PTF11mnb.

\section{Discussion}

The post main-peak photometry of SN 2005bf seems to favor a magnetar as the powering source for that event (Maeda et al. 2007). In the case of PTF11mnb, its slow decline rate instead traces the ${ }^{56} \mathrm{Co}$ decay with no need for adjusting the gamma-ray escape fraction (as in SN 2005bf; see, e.g., Tominaga et al. 2005).

A magnetar cannot be excluded as the main source of the second peak, but ${ }^{56} \mathrm{Ni}$ decay appears to be a simpler explanation. In favor of the magnetar scenario we might consider the shallow bump observed in the redder bands at $\sim 120 \mathrm{~d}$, since a rapidly rotating central engine might produce some variability in the light curve (see, e.g., Nicholl et al. 2016). However, a shallow bump in the redder light curves after peak could be produced by the transition of Fe III ions to Fe II ions as in SNe Ia (see also
Roy et al. 2016). We do not have any signs of circumstellar interaction at late epochs that might explain this bump.

As outlined in the study presented in Taddia et al. (2016a), with our hydrodynamical model we did not intend to explore the entire parameter space, but rather fixed a few parameters based on the previous models of SN 2005bf and the information about PTF11mnb; in particular, we fixed the ejecta mass, helium-poor composition, metallicity of the SN location, and double-peaked ${ }^{56} \mathrm{Ni}$ distribution.

No asymmetry was taken into account when modeling the bolometric light curve. The nebular oxygen line at $6300 \AA$ does suggest a slightly lower degree of asymmetry as compared to the case of SN 2005bf, however the double-peaked ${ }^{56} \mathrm{Ni}$ distribution is likely produced by jets (e.g., see Tominaga et al. 2005 about SN 2005bf).

With PTF11mnb we found indications that some SNe Ic might come from massive $\left(M_{\mathrm{ZAMS}}>85 M_{\odot}\right)$ progenitors characterized by an early emission compatible with the presence of extended material surrounding the progenitor star. The presence of material surrounding the SN at the moment of explosion might be explained in different ways. There are models pointing to massive WR stars that can inflate a tiny quantity of mass, thereby producing a halo structure around the progenitor core (e.g., Ishii et al. 1999). However, in these cases the inflated mass is order of magnitudes lower than what we inferred for PTF11mnb.

Another explanation could be that this material is the result of a strong progenitor eruption. Enhanced mass loss due to eruptions just prior to explosion has been inferred for several SN progenitors (e.g., Ofek et al. 2014) and there are also models predicting these events (Shiode \& Quataert 2014). In the cases of the SE SNe 2006jc (Pastorello et al. 2007) and PTF11qcj (Corsi et al. 2014) eruptions were observed before the final explosion. If we assume that a wind with a velocity of $10^{3} \mathrm{~km} \mathrm{~s}^{-1}$ from the progenitor produced the shell (a value that is typical for WR stars winds), then an extremely high and nonphysical (above $10^{2} M_{\odot} \mathrm{yr}^{-1}$ ) mass-loss rate lasting about $7 \mathrm{~h}$ would be needed to allocate $\sim 0.11 M_{\odot}$ at a distance of $\sim 35 R_{\odot}$. However, in the case of an eruption, the envelope might not be in hydrostatic equilibrium with the star (see Nakar \& Piro 2014; and Piro 2015), therefore in this case we cannot assess its mass with precision, and therefore the estimate of the mass-loss rate would be unreliable. Also, if we assume lower eruption velocities, the mass-loss rate would be more physical (e.g., $\sim 1 M_{\odot} \mathrm{yr}^{-1}$ for about 1 month if we assume a velocity of $\left.10 \mathrm{~km} \mathrm{~s}^{-1}\right)$. According to the models of Shiode \& Quataert (2014), $10^{-3}-1 M_{\odot}$ can be inflated up to $10-100 R_{\odot}$ in a timescale of months to decades, which would be in agreement with the last estimate.

The envelope might also be produced during a common envelope phase in a binary scenario (Chevalier 2012), as it cannot be excluded that our massive progenitor was part of a binary system.

PTF11mnb is the only 2005bf-like SN in the PTF/iPTF sample of $\mathrm{CC} \mathrm{SNe}$. One single case corresponds to an observed fraction of $\sim 0.12 \%$ of the CC SNe and $0.51 \%$ of the SE SNe (including $\mathrm{SNe} \mathrm{IIb}$ ) within (i)PTF. Given their peak luminosity and relatively long rise time, 2005bf-like events should be easier to discover than normal SE SNe. This means they are likely even rarer.

If we assume that only stars with $M_{\text {ZAMS }} \gtrsim 85 M_{\odot}$ can give rise to these events and adopt a normal Salpeter initial mass function (IMF), then we obtain in the single star progenitor scenario that $\sim 4 \%$ of $\mathrm{CC} \mathrm{SNe}$ are formed from stars above that initial mass. Therefore, to match the rate of these events, some 
additional special conditions must occur in $\sim 3 \%$ of these massive stars for a SN 2005bf-like event to occur. It could also be that most of these very massive stars produce failed $\mathrm{SNe}$, as concluded by Smartt (2015).

Ultimately, the similarities between PTF11mnb and SN 2005bf might indicate that the two events share a common powering mechanism, which the analysis of PTF11mnb suggests to be a double-peaked ${ }^{56} \mathrm{Ni}$ distribution. SN 2005bf would be different compared to PTF11mnb after peak due to a larger gamma-ray escape, possibly related to the geometry of the explosion. However, it is not excluded that both SN 2005bf and PTF $11 \mathrm{mnb}$ are powered by a magnetar as proposed by Maeda et al. (2007) for SN 2005bf, with the magnetar fortuitously mimicking the ${ }^{56} \mathrm{Ni}$ decay in the case of PTF11mnb.

\section{Conclusions}

We presented observations for PTF11mnb, a SN Ic whose early and main-peak light curves resemble those of SN 2005bf. PTF11mnb never shows He in its spectra contrary to SN 2005bf.

Its slowly declining light curve suggests that a doubledpeaked ${ }^{56} \mathrm{Ni}$ distribution powers both light-curve peaks with most of the ${ }^{56} \mathrm{Ni}$ located in the center. Based on the large ejecta mass implied by the late-time main peak, the progenitor star of PTF11mnb could have been a massive, possibly single WR star, which was entirely stripped of its hydrogen and helium envelope.

However, a hybrid model with a magnetar powering the main peak and a normal SN explosion powering the first peak (as suggested for SN 2005bf) cannot be excluded. In this case the ejecta mass would be significantly lower.

The early $g$-band light curve emission suggests the presence of an extended envelope surrounding the progenitor of PTF11mnb, as in the case of other massive SN Ic progenitors.

Acknowledgements. We gratefully acknowledge the support from the Knut and Alice Wallenberg Foundation. Based on observations obtained with the Samue Oschin 48-inch Telescope and the 60-inch Telescope at the Palomar Observatory as part of the intermediate Palomar Transient Factory (iPTF) project, a scientific collaboration among the California Institute of Technology, Los Alamos National Laboratory, the University of Wisconsin, Milwaukee, the Oskar Klein Center, the Weizmann Institute of Science, the TANGO Program of the University System of Taiwan, and the Kavli Institute for the Physics and Mathematics of the Universe. The Oskar Klein Centre is funded by the Swedish Research Council. We thank I. Arcavi, A. Horesh, T. Kupfer, D. Levitan, T. Matheson, G Smadja, S. Tendulkar, and D. Xu for their help with the spectral observations and their preliminary reductions. We thank Y. Cao, J. Surace, R. Laher, F. Masci, U. Rebbapragada, and P. Woźniak for their contribution to the iPTF project. This work is partly based on observations made with DOLoRes@TNG. A.G.-Y. is supported by the EU/FP7 via ERC grant No. 725161, the Quantum Universe I-Core program by the Israeli Committee for planning and funding, and the ISF, Minerva and ISF grants, WIS-UK "making connections", and Kimmel and YeS awards. G.S. acknowledges support from the Lyon Institute of Origins under grant ANR-10-LABX-66.

\section{References}

Ahn, C. P., Alexandroff, R., Allende Prieto, C., et al. 2014, ApJS, 211, 17 Anupama, G. C., Sahu, D. K., Deng, J., et al. 2005, ApJ, 631, L125 Arcavi, I., Gal-Yam, A., Kasliwal, M. M., et al. 2010, ApJ, 721, 777 Arnett, W. D. 1982, ApJ, 253, 785

Asplund, M., Grevesse, N., Sauval, A. J., \& Scott, P. 2009, ARA\&A, 47, 481
Bersten, M. C., Benvenuto, O., \& Hamuy, M. 2011, ApJ, 729, 61 Bianco, F. B., Modjaz, M., Hicken, M., et al. 2014, ApJS, 213, 19 Blinnikov, S. I., \& Bartunov, O. S. 1993, A\&A, 273, 106 Blondin, S., \& Tonry, J. L. 2007, ApJ, 666, 1024

Cano, Z. 2013, MNRAS, 434, 1098

Cenko, S. B., Fox, D. B., Moon, D.-S., et al. 2006, PASP, 118, 1396 Chevalier, R. A. 2012, ApJ, 752, L2

Corsi, A., Ofek, E. O., Gal-Yam, A., et al. 2014, ApJ, 782, 42

Dessart, L., Hillier, D. J., Woosley, S., et al. 2015, MNRAS, 453, 2189 Drout, M. R., Soderberg, A. M., Gal-Yam, A., et al. 2011, ApJ, 741, 97 Eldridge, J. J., Fraser, M., Smartt, S. J., Maund, J. R., \& Crockett, R. M. 2013, MNRAS, 436, 774

Elmhamdi, A., Danziger, I. J., Branch, D., et al. 2006, A\&A, 450, 305 Ergon, M., Sollerman, J., Fraser, M., et al. 2014, A\&A, 562, A17 Ergon, M., Jerkstrand, A., Sollerman, J., et al. 2015, A\&A, 580, A142 Folatelli, G., Contreras, C., Phillips, M. M., et al. 2006, ApJ, 641, 1039 Fremling, C., Sollerman, J., Taddia, F., et al. 2016, A\&A, 593, A68 Fukugita, M., Ichikawa, T., Gunn, J. E., et al. 1996, AJ, 111, 1748 Hunter, D. J., Valenti, S., Kotak, R., et al. 2009, A\&A, 508, 371 Ishii, M., Ueno, M., \& Kato, M. 1999, PASJ, 51, 417 Kasen, D., \& Bildsten, L. 2010, ApJ, 717, 245

Kasen, D., Metzger, B. D., \& Bildsten, L. 2016, ApJ, 821, 36 Komatsu, E., Dunkley, J., Nolta, M. R., et al. 2009, ApJS, 180, 330 Law, N. M., Kulkarni, S. R., Dekany, R. G., et al. 2009, PASP, 121, 1395 Liu, Y., \& Modjaz, M. 2014, ArXiv e-prints [arXiv: 1405 . 1437] Lyman, J. D., Bersier, D., \& James, P. A. 2014, MNRAS, 437, 3848 Lyman, J. D., Bersier, D., James, P. A., et al. 2016, MNRAS, 457, 328 Maeda, K., Tanaka, M., Nomoto, K., et al. 2007, ApJ, 666, 1069 Masci, F. J., Laher, R. R., Rebbapragada, U. D., et al. 2017, PASP, 129, 014002 Maund, J. R., Wheeler, J. C., Patat, F., et al. 2007, MNRAS, 381, 201 Milisavljevic, D., Fesen, R. A., Gerardy, C. L., Kirshner, R. P., \& Challis, P. 2010, ApJ, 709, 1343

Modjaz, M., Kirshner, R. P., Blondin, S., Challis, P., \& Matheson, T. 2008, ApJ, 687, L9

Modjaz, M., Liu, Y. Q., Bianco, F. B., \& Graur, O. 2016, ApJ, 832, 108

Monard, L. A. G., Moore, M., \& Li, W. 2005, IAU Circ., 8507, 1

Morozova, V., Piro, A. L., Renzo, M., et al. 2015, ApJ, 814, 63

Nakar, E., \& Piro, A. L. 2014, ApJ, 788, 193

Nicholl, M., Berger, E., Smartt, S. J., et al. 2016, ApJ, 826, 39 Ofek, E. O., Sullivan, M., Shaviv, N. J., et al. 2014, ApJ, 789, 104

Oke, J. B., \& Gunn, J. E. 1982, PASP, 94, 586

Oke, J. B., Cohen, J. G., Carr, M., et al. 1995, PASP, 107, 375

Parrent, J., Branch, D., Troxel, M. A., et al. 2007, PASP, 119, 135

Pastorello, A., Smartt, S. J., Mattila, S., et al. 2007, Nature, 447, 829

Paxton, B., Bildsten, L., Dotter, A., et al. 2011, ApJS, 192, 3

Pettini, M., \& Pagel, B. E. J. 2004, MNRAS, 348, L59

Piro, A. L. 2015, ApJ, 808, L51

Piro, A. L., \& Nakar, E. 2013, ApJ, 769, 67

Prentice, S. J., Mazzali, P. A., Pian, E., et al. 2016, MNRAS, 458, 2973

Rahmer, G., Smith, R., Velur, V., et al. 2008, Proc. SPIE, 7014, 70144

Rau, A., Kulkarni, S. R., Law, N. M., et al. 2009, PASP, 121, 1334

Roy, R., Sollerman, J., Silverman, J. M., et al. 2016, A\&A, 596, A67

Sanders, N. E., Soderberg, A. M., Levesque, E. M., et al. 2012, ApJ, 758, 132

Sanders, N. E., Levesque, E. M., \& Soderberg, A. M. 2013, ApJ, 775, 125

Schlafly, E. F., \& Finkbeiner, D. P. 2011, ApJ, 737, 103

Shiode, J. H., \& Quataert, E. 2014, ApJ, 780, 96

Smartt, S. J. 2015, PASA, 32, e016

Taddia, F., Sollerman, J., Leloudas, G., et al. 2015, A\&A, 574, A60

Taddia, F., Fremling, C., Sollerman, J., et al. 2016a, A\&A, 592, A89

Taddia, F., Sollerman, J., Fremling, C., et al. 2016b, A\&A, 588, A5

Taddia, F., Stritzinger, M. D., Bersten, M., et al. 2017, A\&A, in press, DOI: 10.1051/0004-6361/201730844

Tanaka, M., Kawabata, K. S., Maeda, K., et al. 2009, ApJ, 699, 1119

Taubenberger, S., Pastorello, A., Mazzali, P. A., et al. 2006, MNRAS, 371, 1459

Tominaga, N., Tanaka, M., Nomoto, K., et al. 2005, ApJ, 633, L97

Valenti, S., Taubenberger, S., Pastorello, A., et al. 2012, ApJ, 749, L28

Yaron, O., \& Gal-Yam, A. 2012, PASP, 124, 668

Yoon, S.-C., Woosley, S. E., \& Langer, N. 2010, ApJ, 725, 940 